\title{
Binary Quantum Communication using Squeezed Light: Theoretical and Experimental Frame Work
}

\author{
Ismael S. Desher Alaskary ${ }^{1}$ and R. S. Fyath ${ }^{2}$ \\ ${ }^{1}$ University of Baghdad, Baghdad, Iraq \\ Desher19@yahoo.com \\ ${ }^{2}$ College of Engineering, Alnahrain University, Baghdad, Iraq \\ rsfyath@yahoo.com
}

\section{ABSTRACT}

The aim of this paper is to develop framework to generate squeezed light for binary quantum communication. Both theoretical and experimental models to generate squeezed state using optical parametric amplifier (OPO), which is implemented around $\mathrm{He}-\mathrm{Ne}$ laser, are described in details. The results will be used as a guide line to investigate the performance of squeezed light-based quantum communication over noisy channel and this issue will be presented in accompanying paper [1].

\section{Indexing terms/Keywords}

Squeezed light; Quantum binary communication.

\section{Academic Discipline And Sub-Disciplines} Quantum communication.

\section{SUBJECT CLASSIFICATION}

$90 \mathrm{C} 26$.

\section{TYPE (METHOD/APPROACH)}

Theoretical, simulation, and experimental investigation.

\section{Council for Innovative Research}

\author{
Peer Review Research Publishing System
}




\section{INTRODUCTION}

Quantum state of light lies in the heart of quantum theory. It is represented by a vector in the complex Hilbert space and contained all the information about the possible results of measurement on the system. The quantum system should obey the Heisenberg uncertainty principle. From this point of view, intrinsic random fluctuations have been imposed by the uncertainty principle, which prohibits simultaneous determination of the position and the momentum. On the other hand, the quantum state is called a pure state if it is contained the maximum information about the system otherwise it is a mixed state that combined from two or more pure states [2].

There are three fundamental types of quantum states, Fock, coherent, and squeezed states. Fock states or number states are purely non- classical quantum states. They are used as a basis in quantum information technology such as quantum communication and quantum computation [3]. There is an enormous difficulty for the generation of this type of states but recently many efforts are exerted to generate a source of multi-photon number states [4-6]. Instead, a fainted laser pulse may be, in average, considered as a single photon source according to the Poisson distribution [7]. On the other hand, the fainted pulse is based on a classical light. Therefore, it can generate a single photon but under the conception of classical (coherent) quantum state. Thus, it can not be treated as a non classical state. Coherent states or quasi-classical states are classical quantum states whose dynamics are most closely resembled the classical coherent light. They have the minimum constraint by the uncertainty principle with equally fluctuations in the position and the momentum [8]. Moreover, these states are Gaussian states because their Wigner functions are Gaussian. Thus, the coherent state may be fully described by determining the mean and the variance of the state [9]. The physical implementation of the coherent states is very easy that can be given by a highly stabilized laser operating well above threshold. Thus, the coherent states became important as a tool for connecting quantum and classical world, therefore their name quasi-classical state. Due to these significant properties and reliabilities, the coherent states play a key role in many applications such as quantum communication [10], quantum computation [11], quantum teleportation [12], and quantum repeater [13, 14].

Squeezed states are non-classical states having a quantum noise in one quadrature below the standard quantum limit at the expense of the other while keeping the uncertainty principle hold [15]. In spite of the squeezing in one quadrature, the quantum (squeezed) non-classical states are still having Gaussian shapes [16]. In contrast, of the quantum (coherent) classical state, the generation of the squeezed states is not trivial. Spontaneous parametric down conversion (SPDC) is the most often process used to generate the squeezed state [17]. SPDC is a second-order nonlinear process in which a high frequency photon spontaneously splits in two lower frequency photons, in accordance with the energy conservation law [18]. SPDC is applied to initiate the process in the optical-parametric oscillator (OPO) through a nonlinear crystal. Often, a temperature control is needed to achieve non-critical phase matching (NCPM) inside the nonlinear crystal at the presence of a strong pump beam [19]. There is a strong interest in the squeezed states for quantum communication [20], quantum cryptography [21] and gravitational interferometer [22-24].

Binary quantum communication may be realized using two quantum states that can carry classical information of logic 0 and 1 [25]. Since the states are in general not orthogonal and even they are so, the orthogonality will be rapidly destroyed due to the noise effects that added by the channel during the transmission of the state. Therefore, unavoidable errors will be occurred in the receiver side because of the overlap between the received states. Moreover, the channel is a freespace channel. Therefore, the states are affected by the thermal noise added by the environment and the dissipation. Hence, there is a need to investigate the best performance of discriminating noisy non-orthogonal states [26]. The realization of an optimal quantum receiver with minimum measurement errors is a crucial topic for the effective implementation of quantum communication channel. Receivers based on homodyne detection have great advantages for the discrimination between states in the presence of noise [27]. Moreover, coherent and squeezed states are Gaussian states then homodyne receivers have been demonstrated to represent the optimum Gaussian method for the discrimination of the binary quantum communication $[28,29]$. These issues will be addressed in details in this work.

\section{SQUEEZED STATE}

Define quadrature operators $\hat{q}$ and $\hat{p}$ which are Hermitian operators representing the real and imaginary parts of the annihilation operator. The variances of the quadrature operators are equal to $1 / 2$. Thus, they are symmetric and the minimum uncertainty principle may be allowed for the vacuum and the coherent state after the following relation [30]

$\Delta \hat{q} \Delta \hat{p}=\frac{1}{2}$

So the squeezing may be achieved by squeezing one quadrature at the expense of stretching the other keeping the Heisenberg relation unchanged as shown in Figure 1.

The squeezed vacuum state of a single-mode field is defined by applying the squeezed operator $\hat{S}(\xi)$ on the vacuum state

$|\xi\rangle=\hat{S}(\xi)|0\rangle$

The squeezed operator is a unity operator given by [31]

$\hat{S}(\xi)=\exp \left(\frac{1}{2} \xi \hat{a}^{+2}-\frac{1}{2} \xi^{*} \hat{a}^{2}\right)$

with the annihilation and creation operators $\hat{a}$ and $\hat{a}^{+}$, respectively. Further, $\xi=r \exp (\varnothing)$ is a complex parameter. $r$ is the squeezed parameter varying from 0 to $\infty$ representing the amplitude and $\varnothing$ is the phase defining the squeezing quadrature. The action of the squeezed operator on the annihilation and creation operators can be expressed as [31] 


$$
\begin{aligned}
& \hat{S}^{+}(\xi) \hat{a}^{+} \hat{S}(\xi)=\hat{a} \cosh r-\hat{a}^{+} e^{i \emptyset} \sinh r \\
& \hat{S}^{+}(\xi) \hat{a} \hat{S}(\xi)=\hat{a}^{+} \cosh r-\hat{a} e^{-i \emptyset} \sinh r
\end{aligned}
$$

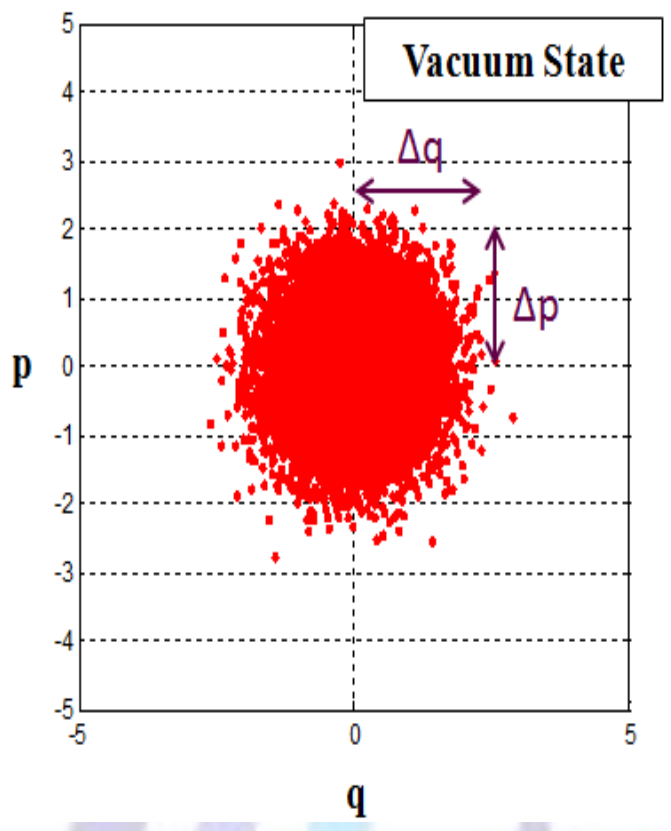

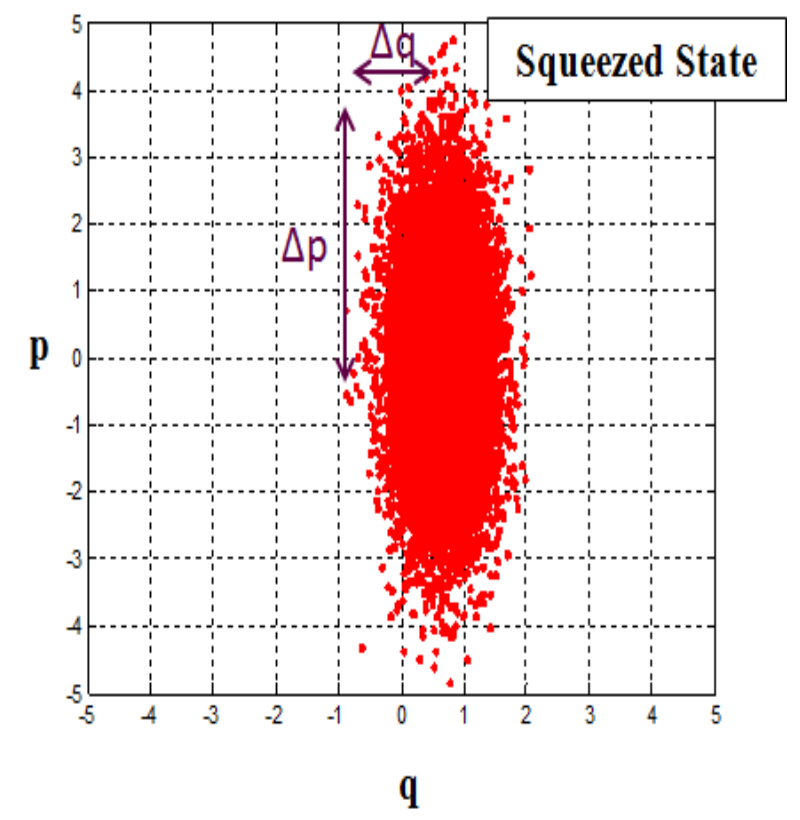

Figure 1: Phase space representation of (a) Vacuum state. (b) Squeezed vacuum state.

The action of the squeezed operator on the quadrature operators in order to evaluate the variance and the mean of a single mode coherent squeezed state [31]

$$
\begin{aligned}
& \left(\Delta q_{s q}\right)^{2}=\frac{1}{2}\left(e^{-2 r} \cos ^{2} \frac{\emptyset}{2}+e^{2 r} \sin ^{2} \frac{\emptyset}{2}\right) \\
& \left(\Delta p_{s q}\right)^{2}=\frac{1}{2}\left(e^{-2 r} \sin ^{2} \frac{\emptyset}{2}+e^{2 r} \cos ^{2} \frac{\emptyset}{2}\right)
\end{aligned}
$$

$\left(\Delta p_{s q}\right)^{2}=\frac{1}{2} e^{2 r}$

This indicates clearly that the variance is squeezed in one quadrature by $e^{-2 r}$ and stretched in the other by $e^{2 r}$. The uncertainty principle $\Delta q_{s q} \Delta p_{s q}=1 / 2$ is hold unchanged. The mean photon number is given by [32]

$\langle\hat{n}\rangle_{s q}=|\alpha|^{2}+\sinh ^{2} r$

Eqn. (7) indicates that

(i) Increasing the squeezed amplitude $r$ will increase the mean photon number.

(ii) When $r=0$, non squeezing, (coherent) state is obtained.

(iii) When $\alpha=0$, the squeezed vacuum state is obtained which is characterized by an average number of photons depends on $r$.

Finally, the Wigner function of the squeezed coherent state along the q quadrature can be evaluated as [32]

$W_{s q}(q, p)=\frac{1}{\pi} \exp \left[-e^{-2 r}\left(q-q_{0}\right)^{2}-e^{2 r}\left(p-p_{0}\right)^{2}\right]$

The squeezed coherent state can also be obtained by shifting the squeezed vacuum state using the displacement operator as shown in Figure 2

$|\alpha, \xi\rangle=\widehat{D}(\alpha) \hat{S}(\xi)|0\rangle$ 

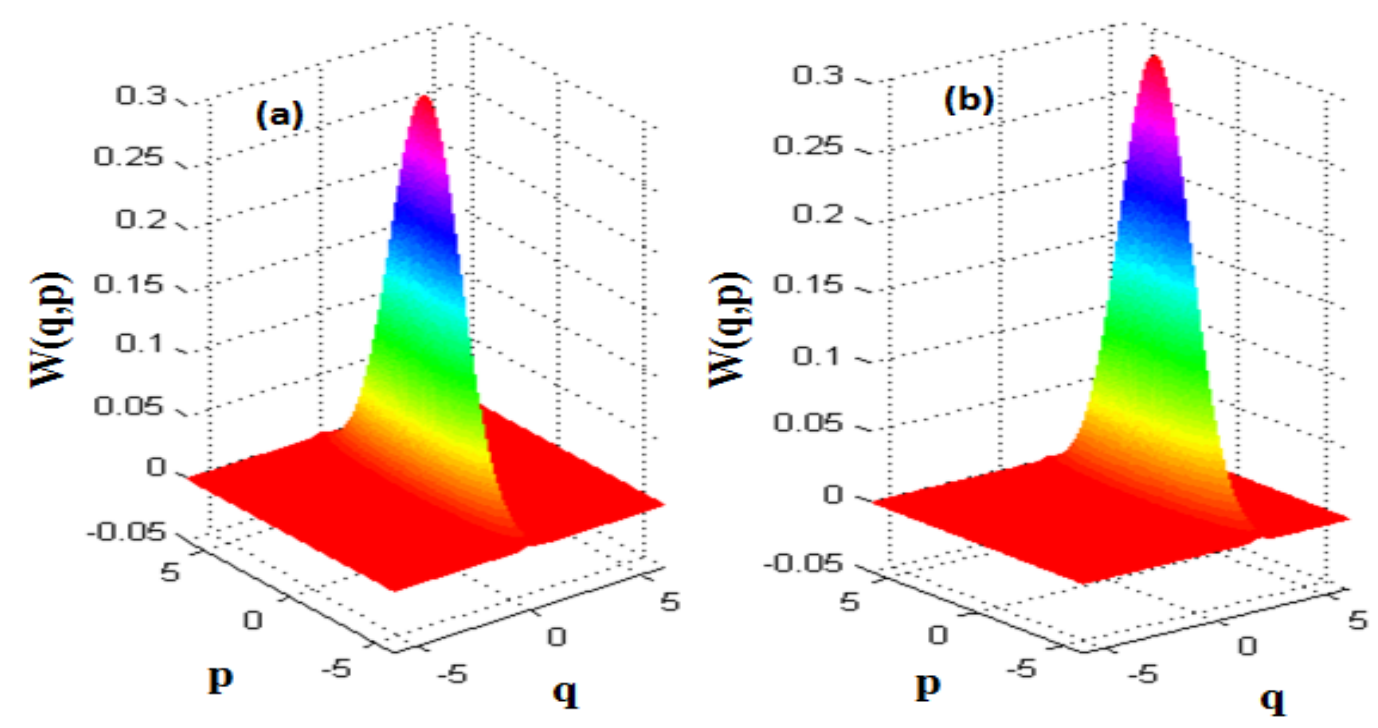

Figure 2: Wigner functions for (a) Squeezed vacuum state. (b) Squeezed coherent state $r=1$.

\section{GENERATION Of SQUEEZED STATE USING OPO: EXPERIMENTAL FRAME WORK}

This section is directed to perform a setup for generating squeezed states of light using OPO. The setup consists of four main parts as shown in Figure 3.

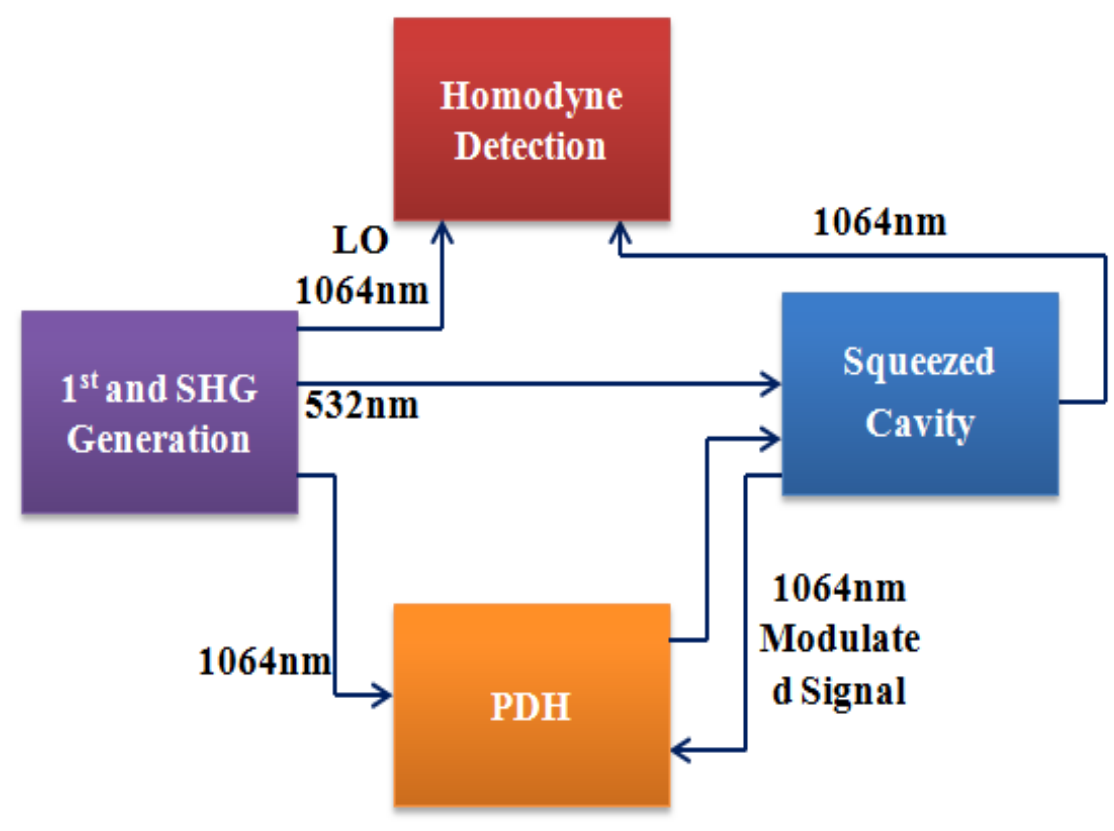

Figure 3: Block diagram of the squeezed setup

(i) Nd-YAG laser is used to generate the first harmonic (1064 nm) and the second harmonic (SHG) at $532 \mathrm{~nm}$. The fundamental laser system is built through a ring cavity.

(ii) Squeezed cavity involves two curved mirrors and a nonlinear crystal.

(iii) A Pound-Drever-Hall (PHD) system controls the cavity length of the OPO.

(iv) Balanced homodyne detection (BHD) is used to detect the squeezed state of light with a strong reference as a local oscillator (LO).

\subsection{First and Second-Harmonic Generation}

This subsection involves two principal parts:

(i) The setup design includes different computations: the cavity dimensions, position of the multiple devices, the spot size of the beam, and the expected power generated by the setup. 
(ii) Experimental setup to generate the first and the second harmonics.

\subsubsection{Preliminary Calculations}

The first and the second harmonics are generated by a ring resonator as shown in Figure 4. It consists of four mirrors: two are planes and the other are curved. A periodically poled lithium niobate (PPLN) crystal is used to generate the second harmonic while the first harmonic is generated using an Nd-YAG rod.

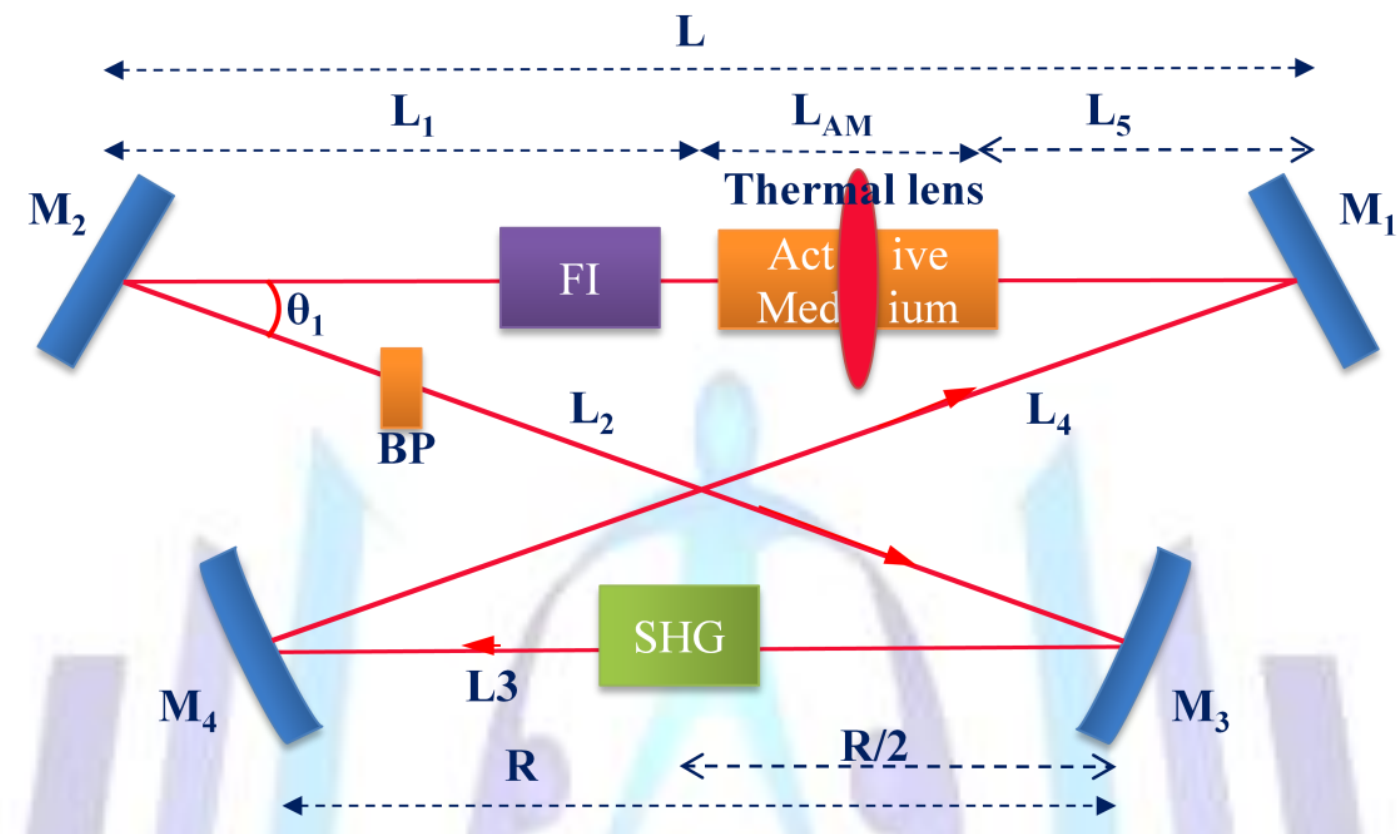

Figure 4: Setup to generate $1064 \mathrm{~nm}$ and $532 \mathrm{~nm}$ wavelengths. $\mathrm{M}_{1}, \mathrm{M}_{4}$ : Plane mirrors. $\mathrm{M}_{2}, \mathrm{M}_{3}$ : Curved mirrors. FI: Faraday Insulator. BP: Brewster plate.

In Figure $4, \theta_{1}=15^{\circ}, L_{1}=220 \mathrm{~mm}, R=300 \mathrm{~mm}, L_{F I}=20 \mathrm{~mm}, L=400 \mathrm{~mm}$, the Brewster plate length $h_{B P}=2.21 \mathrm{~mm}$, $L_{2}=L_{4}=\left(R / 2+L_{1}\right) / \cos \left(\theta_{1}\right)$ and $L_{A M}=60 \mathrm{~mm}$. The starting point is to calculate the refractive indices of the barium borosilicate glass (BK7) and the Nd-YAG rod using Sellmeier equation [33, 34]

$n(\lambda)=\sqrt{\left(A+\frac{B_{1} \lambda^{2}}{\lambda^{2}-C_{1}}+\frac{B_{2} \lambda^{2}}{\lambda^{2}-C_{2}}+\frac{B_{3} \lambda^{2}}{\lambda^{2}-C_{3}}\right)}$

$A, B_{1}, B_{2}, B_{3}, C_{1}, C_{2}$, and $C_{3}$ are the Sellmeier coefficients. $\lambda$ is the wavelength related to the material in $\mu m$. Eqn. (10) is the dispersion equation describing the relation between the refractive index and the wavelength for a given material. Table 1 displays the coefficients of Sellmeier equation for the Nd-YAG rod and the BK7.

\section{Table 1: Sellmeier coefficients of Nd-YAG and BK7.}

\begin{tabular}{|c|c|c|c|c|}
\hline Coefficients & Nd-YAG & 1 \\
\hline A & 1 & 1.03961212 \\
\hline$B_{1}$ & 2.2779 & $6.00069867 \times 10^{-3}$ \\
\hline$C_{1}\left(\mu m^{2}\right)$ & 0.01142 & 0.231792344 \\
\hline$B_{2}$ & 0 & $2.00179144 \times 10^{-2}$ \\
\hline$C_{2}\left(\mu m^{2}\right)$ & 0 & 1.01046945 \\
\hline$B_{3}$ & 0 & $1.03560653 \times 10^{2}$
\end{tabular}

From Table 1 the refractive indices are

(i) $\quad n_{N d}=1.817$ at $1064 \mathrm{~nm}$

(ii) $\quad n_{B K}=1.507$ at $1064 \mathrm{~nm}$. 
Next step, calculate the free spectral range (FSR) of the cavity. Note that the FSR depends on the effective length of the cavity $L_{e f f}$ which related to the ring physical length $L_{\text {ring }}$ [35]

$L_{\text {ring }}=L_{1}+L_{2}+R+L_{3}$

$$
=L_{1}+2 \frac{\frac{R}{2}+L_{1}}{\cos \left(\theta_{1}\right)}+R+L_{5}
$$

$L_{e f f}=L_{\text {ring }}+\left[\frac{h_{B P} n_{B K}}{\sin \left(\theta_{B}\right)}-\frac{h_{B P} \sin \left(2 \theta_{B}\right)}{\sin \left(\theta_{B}\right)}\right]+n_{N d-Y A G}+L_{F I}$

where $\theta_{B}=56.427^{\circ}$ is the Brewster angle. The FSR is given by

$F S R=\frac{c}{2 L_{e f f}}$

The calculation yields: $L_{e f f}=1475 \mathrm{~mm}$ hence $F S R=102 \mathrm{MHz}$. Thus, the frequency spacing between two modes is 102 $\mathrm{MHz}$.

Next, it is fundamental to determine the stability of the cavity for the fundamental transversal mode. TEM $M_{00}$ which has a Gaussian transversal profile and represents a Gaussian beam. This can be derived from Gaussian beam propagation through the resonator under the condition of self-reproduction after one complete round trip. This requires the computing of the round-trip ABCD matrices along the ring resonator. Take the reference plane at the thermal lens in Figure 4 and going from right to left. The matrix form is either lens matrix $M_{l}$ or free space matrix $M_{S}$ as follow [35]

$M_{S}(z)=\left[\begin{array}{ll}1 & z \\ 0 & 1\end{array}\right]$

where $\mathrm{z}$ represents the propagation distance and

$M_{l}(f)=\left[\begin{array}{cc}1 & 0 \\ -\frac{1}{f} & 1\end{array}\right]$

where $\mathrm{f}$ is the focal length of a thin lens. Hence, the round-trip matrix $M_{R T}$ is a product of $M_{i}$ matrices. i refers to the medium in the resonator and each matrix corresponds to a medium where the beam propagates.

$$
\begin{aligned}
M_{R T} & =\left[\begin{array}{ll}
A & B \\
C & D
\end{array}\right] \\
& =\prod_{i=1}^{12} M_{i}
\end{aligned}
$$

\begin{tabular}{|c|c|c|c|}
\hline$M_{1}$ & $M_{2}$ & $M_{3}$ & $M_{4}$ \\
\hline$M_{l}\left(f_{t h}\right)$ & $M_{S}\left(\frac{L_{A M}}{2 n_{N d}}\right)$ & $M_{S}\left(L_{5}\right)$ & $M_{S}\left(L_{4}\right)$ \\
\hline$M_{5}$ & $M_{6}$ & $M_{7}$ & $M_{8}$ \\
\hline$M_{l}\left(\frac{R_{O C} \cos \left(\frac{\theta_{1}}{2}\right)}{2}\right)$ & $M_{S}\left(\frac{R}{2}\right)$ & $M_{l}(G R I I R A)$ & $M_{S}\left(\frac{R}{2}\right)$ \\
\hline$M_{9}$ & $M_{10}$ & $M_{11}$ & $M_{12}$ \\
\hline$M_{l}\left(\frac{R_{O C} \cos \left(\frac{\theta_{1}}{2}\right)}{2}\right)$ & $M_{S}\left(L_{2}\right)$ & $M_{S}\left(L_{1}\right)$ & $M_{S}\left(\frac{L_{A M}}{2 n_{N d}}\right)$ \\
\hline
\end{tabular}

where, A, B, C and D are the matrix coefficients and $M_{i}$ is shown in Table 2.

Table 2: ABCD matrices for the resonator stability.

The stability condition can be guessed from Eqn. (14) [35]

$m=\frac{|A+D|}{2}<1$ 
Since the matrix coefficients are function of the focal length of the thermal lens then the stability, the waist and the inverse radius of curvature depend also on this parameter.

The position of the crystal used to generate the second harmonic should be given at the maximum focusing of the first harmonic. Therefore, there is a need to determine the propagation of the beam along the ring cavity. The start point is to calculate the waist $w$ and the inverse of the radius of curvature $R^{-1}$ of the propagation at the reference plane and then in each point of the cavity as follow

(i) Propagation on the thermal lens

$$
\begin{aligned}
& w_{1}=\sqrt{\frac{\lambda|B|}{\pi} \sqrt{\frac{1}{1-m^{2}}}} \\
& R_{1}^{-1}=\frac{D-A}{2 B n_{N d}}
\end{aligned}
$$

(ii) Propagation at the end of the active medium

$$
\begin{aligned}
& w_{2}(z)=\sqrt{\left(1+z R_{1}^{-1}\right)^{2} w_{1}^{2}+\left(\frac{\lambda z}{\pi n_{N d}}\right)^{2} \frac{1}{w_{1}^{2}}} \\
& R_{2}(z)^{-1}=\frac{{R_{1}}^{-1}\left(1+z R_{1}^{-1}\right)+\left(\frac{\lambda}{\pi n_{N d^{w_{1}}}^{2}}\right)^{2} z}{\left(1+z R_{1}^{-1}\right)^{2}+\left(\frac{z \lambda}{\pi n_{N d^{w_{1}}}{ }^{2}}\right)^{2}}
\end{aligned}
$$

(iii) Propagation from the end of the active medium to $M_{1}$ then to $M_{2}$

$$
\begin{aligned}
& w_{3}(z)=\sqrt{\left[1+z\left(n_{N d} R_{2}\left(z_{1}\right)^{-1}\right)\right]^{2} w_{2}\left(z_{1}\right)^{2}+\left(\frac{\lambda z}{\pi}\right)^{2} \frac{1}{w_{2}\left(z_{1}\right)^{2}}} \\
& R_{3}(z)^{-1}=\frac{\left(n_{N d} R_{2}\left(z_{1}\right)^{-1}\right)\left[1+z\left(n_{N d} R_{2}\left(z_{1}\right)^{-1}\right)\right]+\left(\frac{\lambda}{\pi w_{2}\left(z_{1}\right)^{2}}\right)^{2} z}{\left[1+z\left(n_{N d} R_{2}\left(z_{1}\right)^{-1}\right)\right]^{2}+\left(\frac{z \lambda}{\pi w_{2}\left(z_{1}\right)^{2}}\right)^{2}}
\end{aligned}
$$

where $z_{1}=L_{A M} / 2$

(iv) Propagation up to the SHG crystal

$$
\begin{aligned}
& w_{4}(z)=\sqrt{\left[1+z\left[\frac{-2 R_{3}\left(z_{2}\right)^{-1}}{R_{O C} \cos \left(\frac{\theta_{1}}{2}\right)}\right]\right]^{2} w_{3}\left(z_{2}\right)^{2}+\left(\frac{\lambda z}{\pi}\right)^{2} \frac{1}{w_{3}\left(z_{2}\right)^{2}}} \\
& R_{4}(z)^{-1}=\frac{\left[\frac{-2 R_{3}\left(z_{2}\right)^{-1}}{R_{O C} \cos \left(\frac{\theta_{1}}{2}\right)}\right]\left[1+z\left[\frac{-2 R_{3}\left(z_{2}\right)^{-1}}{R_{O C} \cos \left(\frac{\theta_{1}}{2}\right)}\right]\right]+\left(\frac{\lambda}{\pi w_{3}\left(z_{2}\right)^{2}}\right)^{2} z}{\left[1+z\left[\frac{-2 R_{3}\left(z_{2}\right)^{-1}}{R_{O C} \cos \left(\frac{\theta_{1}}{2}\right)}\right]\right]^{2}+\left(\frac{z \lambda}{\pi w_{3}\left(z_{2}\right)^{2}}\right)^{2}}
\end{aligned}
$$

where $z_{2}=L_{1}+L_{2}$

(v) Propagation from the crystal to $M_{4}$

$$
\begin{aligned}
& w_{5}(z)=\sqrt{\left[1+z R_{4}\left(z_{3}\right)^{-1}\right]^{2} w_{4}\left(z_{3}\right)^{2}+\left(\frac{\lambda z}{\pi}\right)^{2} \frac{1}{w_{4}\left(z_{3}\right)^{2}}} \\
& R_{5}(z)^{-1}=\frac{R_{4}\left(z_{3}\right)^{-1}\left[1+z R_{4}\left(z_{3}\right)^{-1}\right]+\left(\frac{\lambda}{\pi w_{4}\left(z_{3}\right)^{2}}\right)^{2} z}{\left[1+z R_{4}\left(z_{3}\right)^{-1}\right]^{2}+\left(\frac{z \lambda}{\pi w_{4}\left(z_{3}\right)^{2}}\right)^{2}}
\end{aligned}
$$

where $z_{3}=L_{3} / 2$

(vi) Propagation up to the active medium 


$$
\begin{aligned}
& w_{6}(z)=\sqrt{\left[1+z\left[\frac{-2 R_{5}\left(z_{4}\right)^{-1}}{R_{O C} \cos \left(\frac{\theta_{1}}{2}\right)}\right]\right]^{2} w_{5}\left(z_{4}\right)^{2}+\left(\frac{\lambda z}{\pi}\right)^{2} \frac{1}{w_{5}\left(z_{4}\right)^{2}}} \\
& R_{6}(z)^{-1}=\frac{\left[\frac{-2 R_{5}\left(z_{4}\right)^{-1}}{R_{O C} \cos \left(\frac{\theta_{1}}{2}\right)}\right]\left[1+z\left[\frac{-2 R_{5}\left(z_{4}\right)^{-1}}{R_{O C} \cos \left(\frac{\theta_{1}}{2}\right)}\right)\right]+\left(\frac{\lambda}{\pi w_{5}\left(z_{4}\right)^{2}}\right)^{2} z}{\left[1+z\left[\frac{-2 R_{5}\left(z_{2}\right)^{-1}}{R_{O C} \cos \left(\frac{\theta_{1}}{2}\right)}\right]\right]^{2}+\left(\frac{z \lambda}{\pi w_{5}\left(z_{4}\right)^{2}}\right)^{2}}
\end{aligned}
$$

where $z_{4}=L_{3} / 2$

(vii) Propagation in the active medium

$$
\begin{aligned}
& w_{7}(z)=\sqrt{\left(1+z \frac{R_{6}\left(z_{5}\right)^{-1}}{n_{N d}}\right)^{2} w_{6}\left(z_{5}\right)^{2}+\left(\frac{\lambda z}{\pi n_{N d}}\right)^{2} \frac{1}{w_{6}\left(z_{5}\right)^{2}}} \\
& R_{7}(z)^{-1}=\frac{\frac{R_{6}\left(z_{5}\right)^{-1}}{n_{N d}}\left(1+z \frac{R_{6}\left(z_{5}\right)^{-1}}{n_{N d}}\right)+\left(\frac{\lambda}{\left.\pi n_{N d^{w_{6}}\left(z_{5}\right)^{2}}\right)^{2} z}\right.}{\left(1+z \frac{R_{6}\left(z_{5}\right)^{-1}}{n_{N d}}\right)^{2}+\left(\frac{z \lambda}{\pi n_{N d^{w_{6}}\left(z_{5}\right)^{2}}}\right)^{2}}
\end{aligned}
$$

where $z_{5}=L_{4}+L_{5}$

\subsubsection{Experimental Setup to Generate the Second Harmonic}

The experimental setup to generate the first and the second harmonics is shown in Figure 5.

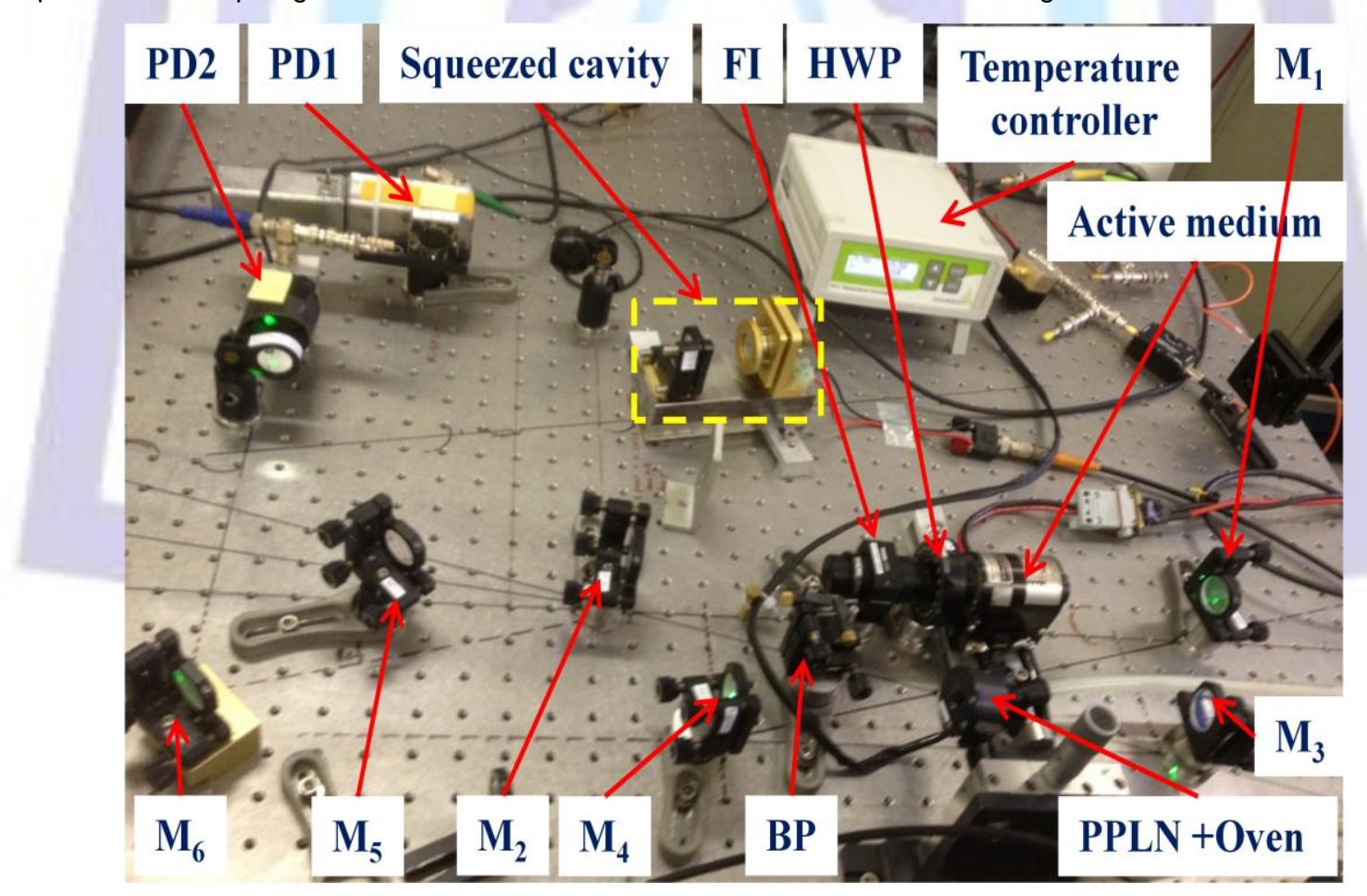

Figure 5: Experimental setup to generate first and second harmonics. $M_{1}, M_{2}, M_{3}, M_{4}, M_{5}, M_{6}$ : Mirrors. PD1, PD2: Photodetectors. BP: Brewster plate. FI: Faraday insulator. HWP: Half wave plate.

The optical devices in Figure 5 have the following specifications:

(i) $\quad \mathrm{M}_{1}$ : Plane mirror with high reflectivity to the first harmonic.

(ii) $\quad \mathrm{M}_{2}$ : Output coupler plane mirror with reflectivity of $93.7 \%$ to the first harmonic.

(iii) $\mathrm{M}_{3}$ : Curved mirror has a radius of curvature $\mathrm{R}_{\mathrm{OC}}=300 \mathrm{~mm}$ with transmission power of $78 \%$ to the second harmonic and high reflectivity to the first harmonic. 
(iv) $\quad \mathrm{M}_{4}$ : Output coupler mirror has a radius of curvature $\mathrm{R}_{\mathrm{OC}}=300 \mathrm{~mm}$ with transmission power of $67 \%$ to the second harmonic and high reflectivity to the first harmonic.

(v) BP: Brewster Plate to minimize the reflection of the p polarized light. It is a (BK7) plate with length of $h_{B P}=$ $22.1 \mathrm{~mm}$.

(vi) Fl: Faraday insulator of $20 \mathrm{~mm}$ is used to prevent the antireflection of the beam into the source.

(vii) PPLN: Nonlinear crystal with 5\% magnesium-oxide MgO doped PPLN. MgO:PPLN offers high-efficiency wavelength conversion. The PPLN has a length of $10 \mathrm{~mm}$ and $1 \mathrm{~mm}$ thickness. The crystal is inserted in a PC10 clip then it is placed in a PV10 oven which is controlled by an OC1 temperature controller.

(viii) Active medium is a Neodymium-doped Yttrium Aluminum Garnet (Nd-YAG) with chemical structure of ( $\mathrm{Nd}: \mathrm{Y}_{3} \mathrm{Al}_{5} \mathrm{O}_{12}$ ). It is a rod of a length of $30 \mathrm{~mm}$ and $2 \mathrm{~mm}$ diameter as shown in Figure 2.11d. Note that during operation, the active medium is equivalent to a thermal lens.

(ix) $\quad \mathrm{PD}_{1}$ and $\mathrm{PD}_{2}$ are used to detect the optical signals by measuring their currents which are converted to voltages with a load resistance of $1 \mathrm{k} \Omega$. To prevent saturation, optical filters are used to attenuate the intensity of the light beams for both PDs. $\mathrm{PD}_{1}$ and $\mathrm{PD}_{2}$ are of silicon types having an efficiency of $0.4 \mathrm{~A} / \mathrm{W}$ at $1064 \mathrm{~nm}$ and 0.23 at $532 \mathrm{~nm}$.

Figure 6 shows the Nd-YAG rod and its equipments.

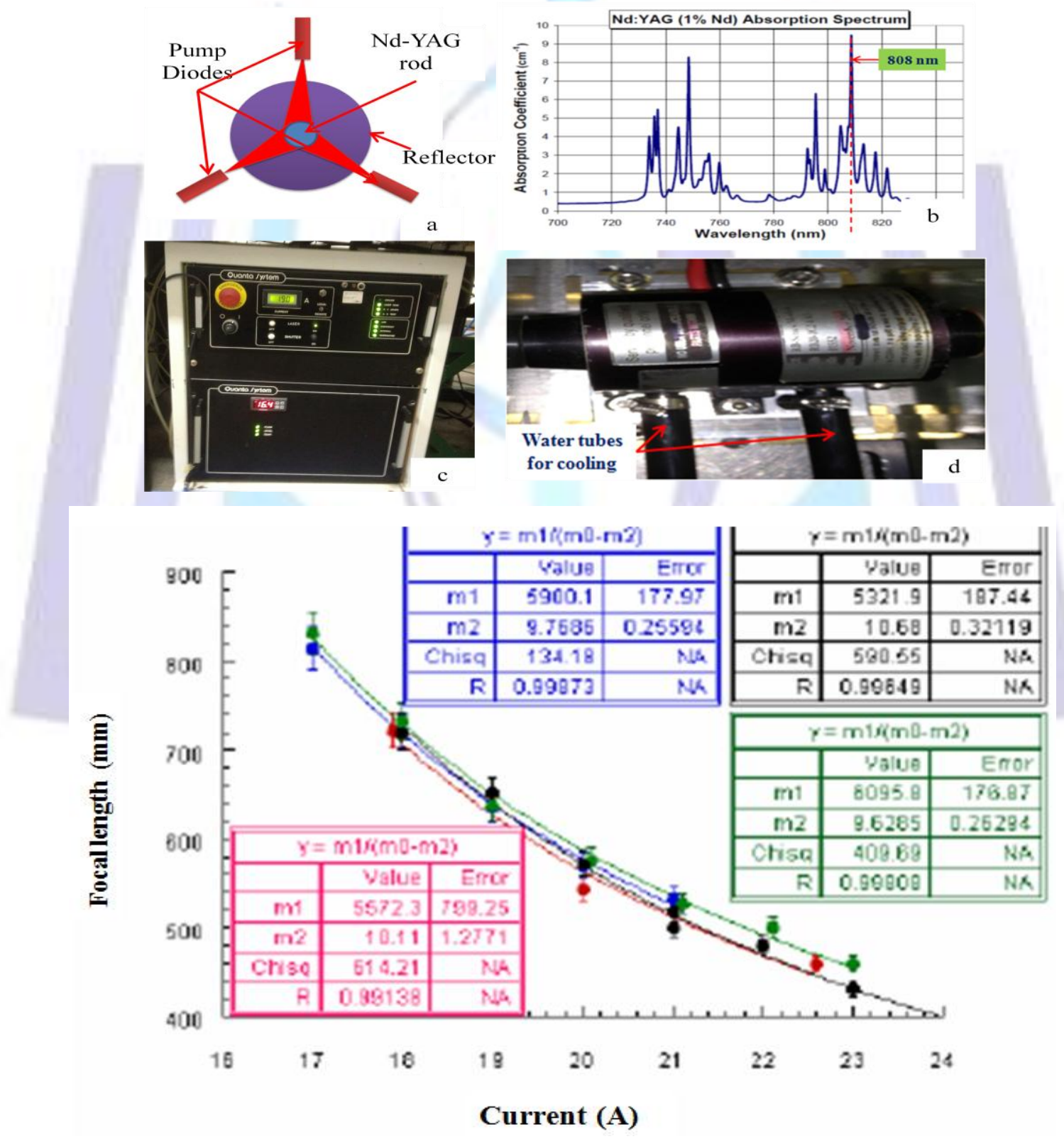

Figure 6: (a) Side pumped laser diodes arranged in slab. (b) Absorption spectrum of the Nd-YAG. (c) Control pupming unit. d: Nd-YAG rod. (e) Thermal lens focal length versus pump current. 
The Nd-YAG rod is side pumped by semiconductor laser diodes at $808 \mathrm{~nm}$. They provide a pump current range up to $24 \mathrm{~A}$ as shown in Figure $6 \mathrm{a}$ and $6 \mathrm{~b}$. A control unit is used to control the pump current and the system cooling which is as important as the rest of the system as shown in Figure 6c.

Without a sufficient cooling, the laser will break down soon. The stability and the efficiency of the laser are quite dependent on cooling. The cooling system is an open-loop cooling system with tap water flowing across the rod. The pump current determines the characteristics of the Nd-YAG laser. As the pump current increases the focal length of the induced thermal lens decreases as show in Figure $6 e$.

In this part of the experiment, the work involves the following steps

(i) Determination of the temperature at which the quasi-phase matching is achieved. This is done by fixing the pump current and increasing the temperature applied on the crystal and marking the second-harmonic power.

(ii) Measuring the first and the second-harmonic powers by increasing the pump current while maintaining the temperature on the PPLN crystal fixes.

(iii) The measures are given by using $\mathrm{PD}_{1}$ and $\mathrm{PD}_{2}$. The photocurrent $I_{P D i}$ detected at the PDi can be written as

$I_{P D i}=R_{i} P_{i \omega}$

where $\mathrm{i}=1$ and 2 which correspond to the first $(1 \omega)$ and the second harmonic $(2 \omega)$, respectively, $R_{i}$ is the responsivity of the $\mathrm{PD}_{i}$ in $\mathrm{A} / \mathrm{W}$ and $P_{i \omega}$ is the optical power incident on the PDs. The incident power $P_{i \omega}$ is calculated by measuring the voltage $V_{i}$ across the detector load resistor $R_{L}=1 \mathrm{k} \Omega$

$P_{i \omega}=\frac{V_{i}}{R_{L} R_{i} T_{f i}}$

where $T_{f i}=0.4$ and 0.23 are the filter power transmission coefficients used to reduce optical-power intensity incident on $P D_{1}$ and $P_{2}$ respectively.

\subsection{Squeezed Cavity Design}

Two curved mirrors are used with their specifications are shown in Table 3.

Table 3: Specifications of $\mathbf{M}_{\mathbf{S 1}}$ and $\mathbf{M}_{\mathbf{S 2}}$.

\begin{tabular}{|c|c|c|c|c|c|}
\hline \multirow{2}{*}{ Mirror } & \multirow{2}{*}{ Roc (mm) } & \multicolumn{2}{|c|}{$R(1064 \mathrm{~nm})$} & \multicolumn{2}{|c|}{$R(532 \mathrm{~nm})$} \\
\hline & & S1 & S2 & S1 & S2 \\
\hline $\mathbf{M}_{\mathbf{S 1}}$ & 25 & AR & $93 \%$ & AR & AR \\
\hline$M_{S 2}$ & 10 & $99 \%$ & AR & HR & - \\
\hline
\end{tabular}

These mirrors should form a squeezed cavity of length $L_{s q}$ as shown in Figure 7. A lithium niobate doped with $5 \%$ magnesium oxide $\mathrm{MgO}\left(\mathrm{Mg}-\mathrm{LNBO}_{3}\right)$ is placed inside the cavity. The ensemble forms an OPO to generate the squeezed light after pumping by the second-harmonic beam.

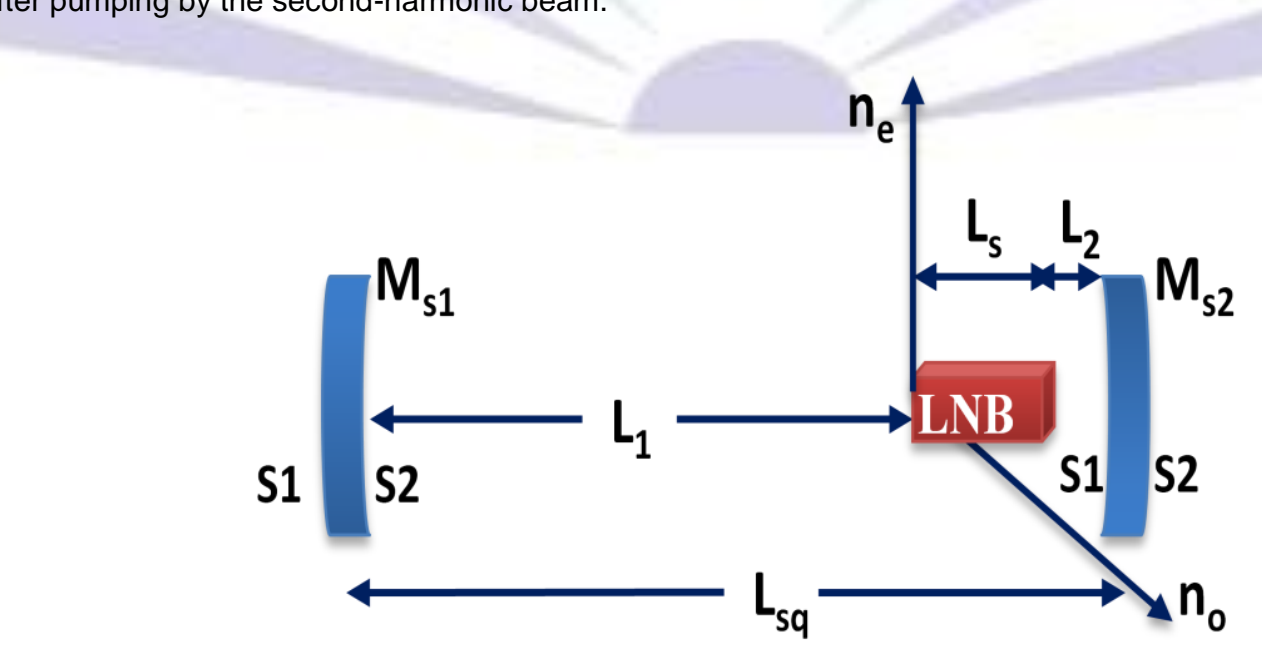

Figure 7: Squeezed setup. $M_{s 1}, M_{s 2}$ : Mirrors. $L_{1}=20 \mathrm{~mm}, L_{2}=3 \mathrm{~mm}$ and $L_{s}=10 \mathrm{~mm}$. 


\subsubsection{Calculation of the Two Refractive Indices}

The crystal is $2 \times 2 \times 10 \mathrm{~mm}, \mathrm{X}$ cut, $\theta=90^{\circ}, \varphi=0$ from Covesion Ltd. [36]. Its two faces are both anti-reflected coating at 1064 $\mathrm{nm}$ and $532 \mathrm{~nm}$. It is a nonlinear crystal with two refractive indices, extraordinary $\mathrm{n}_{\mathrm{e}}$ and ordinary $\mathrm{n}_{\mathrm{o}}$, which are calculated by Sellmeier equation as follow [37]

$n_{e, o}(\lambda, T)=\sqrt{A_{1}+\frac{A_{2}+B_{1} f(T)}{\lambda^{2}-\left[A_{3}+B_{2} f(T)\right]^{2}}+B_{3} f(T)+A_{4} \lambda^{2}}$

where $A_{1}, A_{2}, A_{3}, A_{4}, B_{1}, B_{2}$ and $B_{3}$ are Sellemeier coefficients given in Table $4, \lambda$ is the wavelength of the laser light in $\mu m$ and $f(T)$ is expressed as

$f(T)=(T-24.5)(T+570.5)$

Note that there are two wavelength, $\lambda_{1}$ and $\lambda_{2}$ representing the first and the second harmonics, respectively. The refractive index in Eqn. (25) depends on the wavelength and the temperature because the process to achieve phase matching through the $\mathrm{Mg}-\mathrm{LNBO}_{3}$ is the NCPM.

Table 4: Sellmeier coefficents for $\mathrm{Mg}-\mathrm{LNBO}_{3}$.

\begin{tabular}{|c|c|c|}
\hline Coefficients & $n_{o}$ & $n_{e}$ \\
\hline $\mathrm{A}_{1}$ & 4.9048 & 4.5820 \\
\hline $\mathrm{A}_{2}\left(\mu m^{2}\right)$ & 0.11775 & 0.09921 \\
\hline $\mathrm{A}_{3}(\mu m)$ & 0.21802 & 0.2109 \\
\hline $\mathrm{A}_{4}\left(\mu m^{-2}\right)$ & 0.027153 & 0.02194 \\
\hline $\mathrm{B}_{1}\left(\mu m^{2} /{ }^{\circ} \mathrm{C}\right)$ & $2.2314 \times 10^{-8}$ & $5.2716 \times 10^{-8}$ \\
\hline $\mathrm{B}_{2}\left(\mu m /{ }^{\circ} \mathrm{C}\right)$ & $-2.9671 \times 10^{-8}$ & $-4.9143 \times 10^{-8}$ \\
\hline $\mathrm{B}_{3}\left({ }^{\circ} \mathrm{C}^{-1}\right)$ & $2.1429 \times 10^{-8}$ & $2.2971 \times 10^{-8}$ \\
\hline
\end{tabular}

\subsubsection{Free Spectral Range and Squeezed Resonator Stability}

First, compute the effective length and then the $F S R_{s q}$

$L_{e f f, s q}=L_{1}+L_{2}+n_{o, \lambda_{1}} L_{s}$

The $F S R_{s q}$ is computed from Eqn. (12).

Next, In order to test the stability of the squeezed resonator, one should start to compute the $(A B C D)_{s q}$ matrix of Figure 8 . The reference plane is taken at the mirror $M_{s 1}$ and using Eqns. (13a) and (13b) for the space and lens matrices, respectively. Thus, the round-trip matrix for the squeezed cavity may be written as a product of all matrices elements

$M_{R T, s q}=\prod_{i=1}^{8} M_{i}$

where the $M_{i}$ matrices are described in Table 5. Thus, the stability may be calculated from Eqn. (15).

Table 5: $(A B C D)_{s q}$ matrices for the resonator stability.

\begin{tabular}{|c|c|c|c|}
\hline$M_{\mathbf{1}}$ & $\boldsymbol{M}_{\mathbf{2}}$ & $\boldsymbol{M}_{\mathbf{3}}$ & $\boldsymbol{M}_{\mathbf{4}}$ \\
\hline $\boldsymbol{M}_{\boldsymbol{l}}\left(\frac{\boldsymbol{R}_{\mathbf{1}}}{\mathbf{2}}\right)$ & $M_{S}\left(L_{1}\right)$ & $M_{S}\left(\frac{L_{S}}{n_{o, \lambda_{1}}}\right)$ & $M_{S}\left(L_{2}\right)$ \\
\hline \multicolumn{3}{|c|}{} & $\boldsymbol{M}_{\mathbf{7}}$ \\
\hline $\boldsymbol{M}_{\mathbf{5}}$ & $\boldsymbol{M}_{\mathbf{6}}$ & $M_{S}\left(\frac{L_{s}}{n_{o, \lambda_{1}}}\right)$ & $M_{\mathbf{8}}$ \\
\hline $\boldsymbol{M}_{\boldsymbol{l}}\left(\frac{\boldsymbol{R}_{\mathbf{2}}}{\mathbf{2}}\right)$ & $M_{S}\left(L_{2}\right)$ & & $\left.M_{1}\right)$ \\
\hline
\end{tabular}

\subsubsection{First-Harmonic Propagation}

The start point is to calculate the propagation at each medium 
(i) At the reference plane

$w_{1 s q}\left(L_{1}\right)=\sqrt{\frac{\left|B_{2 s q}\right| \lambda_{1}}{\pi} \sqrt{\frac{1}{1-m_{s q}^{2}}}}$

$R_{1 s q}{ }^{-1}\left(L_{1}\right)=\frac{D_{s q}-A_{s q}}{2 B_{s q}}$

where $A_{2 s q}, B_{2 s q}, C_{2 s q}$ and $D_{2 s q}$ are the coefficient of the squeezed round-trip matrix $M_{T R 2, s q}$ and $m_{s q}$ is the stability condition for the squeezed cavity which is given by

$m_{s q}=\frac{A_{s q}+D_{s q}}{2}$

(ii) From the reference plane to the crystal

$w_{2 s q}(z)=\sqrt{\left[1+z R_{1 s q}\left(L_{1}\right)^{-1}\right]^{2} w_{1 s q}\left(L_{1}\right)^{2}+\left(\frac{\lambda z}{\pi}\right)^{2} \frac{1}{w_{1 s q}\left(L_{1}\right)^{2}}}$

$R_{2 s q}(z)^{-1}=\frac{R_{1 s q}\left(L_{1}\right)^{-1}\left[1+z R_{1 s q}\left(L_{1}\right)^{-1}\right]+\left(\frac{\lambda}{\pi w_{1 s q}\left(L_{1}\right)^{2}}\right)^{2} z}{\left[1+z R_{1 s q}\left(L_{1}\right)^{-1}\right]^{2}+\left(\frac{z \lambda}{\pi w_{1 s q}\left(L_{1}\right)^{2}}\right)^{2}}$

(iii) Inside the crystal

$w_{3 s q}(z)=\sqrt{\left[1+z \frac{R_{2 s q}\left(L_{1}\right)^{-1}}{n_{o, \lambda_{1}}}\right]^{2} w_{2 s q}\left(L_{1}\right)^{2}+\left(\frac{\lambda z}{\pi n_{o, \lambda_{1}}}\right)^{2} \frac{1}{w_{2 s q}\left(L_{1}\right)^{2}}}$

$R_{3 s q}(z)^{-1}=\frac{\frac{R_{2 s q}\left(L_{1}\right)^{-1}}{n_{o, \lambda_{1}}}\left[1+z \frac{R_{2 s q}\left(L_{1}\right)^{-1}}{n_{o, \lambda_{1}}}\right]+\left(\frac{\lambda}{\pi n_{o, \lambda_{1}} w_{2 s q}\left(L_{1}\right)^{2}}\right)^{2} z}{\left[1+z \frac{R_{2 s q}\left(L_{1}\right)^{-1}}{n_{o, \lambda_{1}}}\right]^{2}+\left(\frac{z \lambda}{\pi n_{o, \lambda_{1}} w_{2 s q}\left(L_{1}\right)^{2}}\right)^{2}}$

(iv)

From crystal to air

$w_{4 s q}(z)=\sqrt{\left[1+z\left(n_{o, \lambda_{1}} R_{3 s q}\left(L_{s}\right)^{-1}\right)\right]^{2} w_{3 s q}\left(L_{s}\right)^{2}+\left(\frac{\lambda z}{\pi}\right)^{2} \frac{1}{w_{3 s q}\left(L_{s}\right)^{2}}}$

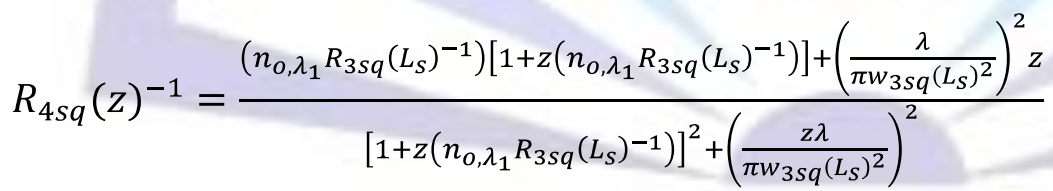

\subsubsection{Second-Harmonic Propagation}

Take the reference plane at the mirror $M_{S 2}$, then the radius of curvature of the beam is the same as that of the mirror $M_{S 2}$.

(i) At $\mathrm{M}_{\mathrm{S} 2}$, the radius of curvature $R_{11}$ is $-10 \mathrm{~mm}$.

(ii) From $\mathrm{M}_{\mathrm{S2}}$ to the crystal

$w_{22}\left(w_{11}, z_{1}\right)=\sqrt{\left(1+z_{1} R_{11}{ }^{-1}\right)^{2} w_{11}{ }^{2}+\left(\frac{\lambda_{2} z_{1}}{\pi}\right)^{2} w_{11}{ }^{-2}}$

$R_{22}{ }^{-1}\left(w_{11}, z_{1}\right)=\frac{R_{11}{ }^{-1}\left(1+z_{1} R_{11}{ }^{-1}\right)+\left(\frac{\lambda_{2}}{\pi w_{11}{ }^{2}}\right)^{2} z_{1}}{\left(1+z_{1} R_{11}{ }^{-1}\right)^{2}+\left(\frac{\lambda_{2} z_{1}}{\pi w_{11}}\right)^{2}}$

(iii) Propagation inside the crystal 


$$
\begin{aligned}
& w_{33}\left(w_{11}, z_{1}\right)=\sqrt{\left[1+z_{1}\left(\frac{R_{22}{ }^{-1}\left(w_{11}, L_{2}\right)}{n_{o, \lambda_{2}}}\right)\right]^{2} w_{22}{ }^{2}\left(w_{11}, L_{2}\right)+\left(\frac{\lambda_{2} z_{1}}{\pi n_{o, \lambda_{2}}}\right)^{2} w_{22}{ }^{-2}\left(w_{11}, L_{2}\right)} \\
& R_{33}{ }^{-1}\left(w_{11}, z_{1}\right)=\frac{\left(\frac{R_{22}{ }^{-1}\left(w_{11}, L_{2}\right)}{n_{o, \lambda_{2}}}\right)\left(1+z_{1}\left(\frac{R_{22}{ }^{-1}\left(w_{11}, L_{2}\right)}{n_{o, \lambda_{2}}}\right)\right)+\left(\frac{\lambda_{2}}{\pi n_{o, \lambda_{2}} w_{22}{ }^{2}\left(w_{11}, L_{2}\right)}\right)^{2} z_{1}}{\left(1+z_{1}\left(\frac{R_{22}{ }^{-1}\left(w_{11}, L_{2}\right)}{n_{o, \lambda_{2}}}\right)\right)^{2}+\left(\frac{\lambda_{2 z_{1}}}{\pi n_{o, \lambda_{2}} w_{22}{ }^{2}\left(w_{11}, L_{2}\right)}\right)^{2}}
\end{aligned}
$$

(iv) Propagation towards the mirror $\mathrm{M}_{\mathrm{S} 1}$ through free space

$$
\begin{aligned}
& w_{44}\left(w_{11}, z_{1}\right)=\sqrt{\left[1+z_{1}\left(n_{o, \lambda_{2}} R_{33}^{-1}\left(w_{11}, L_{S}\right)\right)\right]^{2} w_{33}{ }^{2}\left(w_{11}, L_{S}\right)+\left(\frac{\lambda_{2} z_{1}}{\pi}\right)^{2} w_{33}{ }^{-2}\left(w_{11}, L_{S}\right)} \\
& R_{44}{ }^{-1}\left(w_{11}, z_{1}\right)=\frac{\left(n_{o, \lambda_{2} R_{33}}{ }^{-1}\left(w_{11}, L_{S}\right)\right)\left(1+z_{1}\left(n_{o, \lambda_{2}} R_{33}{ }^{-1}\left(w_{11}, L_{S}\right)\right)\right)+\left(\frac{\lambda_{2}}{\pi w_{33}{ }^{2}\left(w_{11}, L_{S}\right)}\right)^{2} z_{1}}{\left(1+z_{1}\left(n_{o, \lambda_{2}} R_{33}{ }^{-1}\left(w_{11}, L_{S}\right)\right)\right)^{2}+\left(\frac{\lambda_{2} z_{1}}{\pi w_{33}{ }^{2}\left(w_{11}, L_{S}\right)}\right)^{2}}
\end{aligned}
$$

(v) At the mirror $\mathrm{M}_{\mathrm{S} 1}$

$M_{S 1}$ is a thick mirror with radius of curvature $R_{1}=25 \mathrm{~mm}$ and a focal length of

$f_{M_{S 1}}=\frac{1}{\left(n_{B K 7, \lambda_{2}}-1\right)\left(\frac{-1}{R_{1}}\right)}$

Then the propagation from $M_{S 1}$ through free space yields

$$
\begin{aligned}
& w_{55}\left(w_{11}, z_{1}\right)=\sqrt{\left[1+z_{1}\left(\frac{-1}{f_{M_{S 1}}}+R_{44}^{-1}\left(w_{11}, L_{1}\right)\right)\right]^{2} w_{44}^{2}\left(w_{11}, L_{1}\right)+\left(\frac{\lambda_{2} z_{1}}{\pi}\right)^{2} w_{44}{ }^{-2}\left(w_{11}, L_{1}\right)} \\
& R_{55}{ }^{-1}\left(w_{11}, z_{1}\right)=\frac{\left(\frac{-1}{f_{M_{S 1}}}+R_{44}{ }^{-1}\left(w_{11}, L_{1}\right)\right)\left(1+z_{1}\left(\frac{-1}{f_{M_{S 1}}}+R_{44}{ }^{-1}\left(w_{11}, L_{1}\right)\right)\right)+\left(\frac{\lambda_{2}}{\pi w_{44}{ }^{2}\left(w_{11}, L_{1}\right)}\right)^{2} z_{1}}{\left(1+z_{1}\left(\frac{-1}{f_{M_{S 1}}}+R_{44}{ }^{-1}\left(w_{11}, L_{1}\right)\right)\right)^{2}+\left(\frac{\lambda_{2} z_{1}}{\pi w_{44}{ }^{2}\left(w_{11}, L_{1}\right)}\right)^{2}}
\end{aligned}
$$

The waist of the second harmonic is calculated from the waist of the first harmonic for the OPO by the formula [38]

$w_{\lambda 2}=w_{\lambda 1} \frac{1}{\sqrt{2}} \sqrt{\frac{n_{e, \lambda 2}}{n_{o, \lambda 1}}}$

Next, calculate the threshold power required for the OPO. The threshold intensity is given by [39]

$I_{t h}=\left(\frac{n_{e, \lambda 2}}{2 d^{2} Z}\right)\left(\frac{n_{o, \lambda 1} \lambda_{1}}{2 \pi L_{s}}\right)^{2}\left[\frac{1}{2}\left(\frac{1-R_{o c}}{2}+\frac{1-R_{i c}}{2}+\Delta\right)\right]^{2}$

where $Z=1 /\left(\varepsilon_{o} c\right)=377 \Omega$. Since, the pump beam is a single mode Gaussian beam with waist $w_{\lambda 2}$, then, the threshold power for a Gaussian beam is given by [81]

$P_{t h}=\frac{1}{2}\left(\pi w_{\lambda 2}^{2}\right) I_{t h}$

\subsubsection{Generation of Squeezed Light}

The generation of the squeezed light is achieved inside the nonlinear crystal by SPDC. SPDC is a wave mixing process in which the pump decay into two beams at lower frequency namely signal and idler. The crystal is pumped by the second harmonic of a frequency $\omega_{p}$ and some photons of the pump are converted into pairs of identical photons of frequency $\omega_{p} / 2$. The Hamiltonian of this process is given by [40]

$\widehat{H}=\hbar \omega \hat{a}^{+} \hat{a}+\hbar \omega_{p} \hat{b}^{+} \hat{b}+i \hbar \chi^{(2)}\left(\hat{a}^{2} \hat{b}^{+}-\hat{a}^{+2} \hat{b}\right)$

where $\hat{a}, \hat{a}^{+}$and $\hat{b} \hat{b}^{+}$are the annihilation and creation operators of the signal and pump fields respectively and $\chi^{(2)}$ is the second-order susceptibility. The first and the second terms, in Eqn. (42), represent the energy of the signal and the pump, respectively. The third term represents the interaction between the states. However, the pump is a coherent strong field 
that have the state $\left|\beta_{p} e^{-i \omega_{p} t}\right\rangle$. Thus, $\beta_{p} e^{-i \omega_{p} t}$ and $\beta_{p}{ }^{*} e^{i \omega_{p} t}$ correspond to $\hat{b}$ and $\hat{b}^{+}$respectively. Hence, the Hamiltonian in Eqn. (42) becomes

$$
\begin{aligned}
\widehat{H} & =\hbar \omega \hat{a}^{+} \hat{a}+i \hbar \chi^{(2)}\left(\hat{a}^{2} \beta_{p}{ }^{*} e^{i \omega_{p} t}-\hat{a}^{+2} \beta_{p} e^{-i \omega_{p} t}\right) \\
& =\hbar \omega \hat{a}^{+} \hat{a}+i \hbar\left(\eta^{*} \hat{a}^{2} e^{i \omega_{p} t}-\eta \hat{a}^{+2} e^{-i \omega_{p} t}\right)
\end{aligned}
$$

where $\eta=\chi^{(2)} \beta_{p}$. The constant term is dropped because it is irrelevant to the system. Now, transform to the interaction picture yields

$\widehat{H}=i \hbar\left(\eta^{*} \hat{a}^{2} e^{i\left(\omega_{p}-2 \omega_{s}\right) t}-\eta \hat{a}^{+2} e^{-i\left(\omega_{p}-2 \omega_{s}\right) t}\right)$

Since the generated signal has a frequency of $\omega_{s}=\omega_{p} / 2$ then

$\widehat{H}=i \hbar\left(\eta^{*} \hat{a}^{2}-\eta \hat{a}^{+2}\right)$

Using the associated evolution operator $U_{I}(t, 0)$ yields

$$
\begin{aligned}
U_{I}(t, 0) & =\exp \left(\frac{-i \widehat{H}_{I} t}{\hbar}\right) \\
& =\exp \left(\eta^{*} t \hat{a}^{2}-\eta t \hat{a}^{+2}\right)
\end{aligned}
$$

Assuming $\xi=2 \eta t$ then Eqn. (46) has an identical form to the squeezed operator of Eqn. (3).

\subsubsection{Squeezed and Anti-squeezed Variance}

Spontaneous parametric down conversion (SPDC) process is used to generate a squeezed state via an OPO. The Hamiltonian of the OPO is given by [40]

$\widehat{H}=K^{*} \hat{a}^{+} \hat{a}^{+}-K \hat{a} \hat{a}$

where $K=i \hbar \eta$ is a complex constant depends on the nonlinearity and the pump intensity of the OPO as in Eqn. (46). The equation of motion of the OPO is described by [41]

$\dot{\hat{a}}=E \hat{a}^{+}-\gamma \hat{a}+\sqrt{2 \gamma_{i c s 1}} \hat{a}_{b}{ }^{+}+\sqrt{2 \gamma_{l}} \Delta \hat{a}_{l}+\sqrt{2 \gamma_{o c s 2}} \Delta \hat{a}_{c}$

$\dot{\hat{a}}^{+}=E^{*} \hat{a}-\gamma \hat{a}^{+}+\sqrt{2 \gamma_{i c s 1}} \hat{a}_{b}{ }^{+}+\sqrt{2 \gamma_{l}} \Delta \hat{a}_{l}^{+}+\sqrt{2 \gamma_{o c s 2}} \Delta \hat{a}_{c}^{+}$

where $\gamma_{i c s 1}=\left(1-R_{S 1}\right) / 2 T_{R T}$, and $\gamma_{o c s 2}=\left(1-R_{S 2}\right) / 2 T_{R T}$ are the cavity decay rates due to the input and output mirrors reflectivities, $T_{R T}$ is the round trip time, $\gamma_{l}=\Delta / T_{R T}$ is the intra-cavity losses of the OPO, $\Delta$ is the internal loss for a single pass and $\gamma=\gamma_{i c s 1}+\gamma_{o c s 2}+\gamma_{l}$ is the total cavity losses. $\hat{a}_{b}$ represents the signal beam, where $\Delta \hat{a}_{c}$ and $\Delta \hat{a}_{l}$ represent the vacuum fluctuations associated with the losses. Take the Fourier transform (FT) of Eqns. (47a) and (48b) yields

$i \Omega \hat{q}=(\operatorname{Re}[E]-\gamma) \hat{q}+\operatorname{Im}[E] \hat{p}+\sqrt{2 \gamma_{i c s 1}} \hat{q}_{b}+\sqrt{2 \gamma_{l}} \Delta \hat{q}_{l}+\sqrt{2 \gamma_{o c s 2}} \Delta \hat{q}_{c}$

$i \Omega \hat{p}=\operatorname{Im}[E] \hat{p}+(\operatorname{Re}[E]+\gamma) \hat{p}++\sqrt{2 \gamma_{i c s 1}} \hat{p}_{b}+\sqrt{2 \gamma_{l}} \Delta \hat{p}_{l}+\sqrt{2 \gamma_{o c s 2}} \Delta \hat{p}_{c}$

where $\Omega$ is the detection frequency and all $\hat{q}$ and $\hat{p}$ are function of $\Omega$. $\operatorname{Re}[E]$ and $\operatorname{Im}[E]$ denote the real and imaginary parts of the complex number $E$, respectively. Therefore, the spectral density of the noise quadrature components represented by its squeezed and anti-squeezed variances can be calculated from [40]

$\left(\Delta q_{s q}(\Omega)\right)^{2}=\left\langle\hat{q}_{1}^{*}(\Omega) \hat{q}_{1}(\Omega)\right\rangle$

$\left(\Delta p_{\text {anti_sq }}(\Omega)\right)^{2}=\left\langle\left(\hat{p}_{1}(\Omega)\right)^{*}\left(\hat{p}_{1}(\Omega)\right)\right\rangle$

where

$\hat{q}_{1}(\Omega)=\sqrt{2 \gamma_{c}} \hat{q}(\Omega)-\hat{q}_{c}(\Omega)$

Hence the noise variance at the OPO output is [42]

$\left(\Delta q_{s q}(\Omega)\right)^{2}=\frac{1}{2}-\eta_{e s c} \eta_{\text {det }} \eta_{\text {hom }} \frac{2 \sqrt{P / P_{t h}}}{\left(\frac{\Omega}{\gamma}\right)^{2}+\left(1+\sqrt{P / P_{t h}}\right)^{2}}$ 
$\left(\Delta p_{\text {anti_sq }}(\Omega)\right)^{2}=\frac{1}{2}+\eta_{\text {esc }} \eta_{\text {det }} \eta_{\text {hom }} \frac{2 \sqrt{P / P_{t h}}}{\left(\frac{\Omega}{\gamma}\right)^{2}+\left(1-\sqrt{P / P_{t h}}\right)^{2}}$

where $\eta_{\text {det }}$ the efficiency of the photodetectors, $\eta_{\text {hom }}$ the efficiency of the homodyne detection and $\eta_{\text {esc }}$ is the escape efficiency, $\mathrm{P}$ and $P_{t h}$ are the pump and threshold powers.

The most important factor in Eqns. (52a) and (52b) is the escape efficiency $\eta_{\text {esc }}=\gamma_{\text {ocs } 2} / \gamma$ because by increasing this factor, the losses inside the cavity is reduced. Obviously, the escape efficiency can be increased by reducing the reflectivity of the OPO front face. However, this is at the expense of a higher OPO threshold. Thus, a further increase in the escape efficiency is only feasible with a more efficient SHG source or more powerful pump laser.

Now, for power reflectivities of 0.90 and 0.99 for $\mathrm{M}_{\mathrm{S} 1}$ and $\mathrm{M}_{\mathrm{S} 2}$, respectively, and a round trip time $T_{R T}=1 / F S R=3.03 \times$ $10^{-4} \mu \mathrm{s}$, the power spectral losses are: $\gamma_{o c s 1}=164.948 \mathrm{~Hz}, \gamma_{o c s 2}=16.495 \mathrm{~Hz}, \gamma_{l}=0.005 / T_{R T}=16.495 \mathrm{~Hz}$ with internal loss $\Delta=0.005$. Therefore the overall losses $\gamma=197.938 \mathrm{~Hz}$. Hence the escape efficiency $\eta_{\text {esc }}=0.833$. On the other hand, for high-homodyne efficiency $\eta_{\text {hom }}=0.99$ and detection $\eta_{\text {det }}=0.99$. The threshold power $\mathrm{P}_{\mathrm{th}}=1 \mathrm{~mW}$.

The quadrature variance is squeezed from the vacuum noise by the subtracted term in Eqn. (52a) and stretched by the added term in Eqn. (52b). Moreover, Multiple parameters determine the degree of squeezing, the frequency $\Omega$, the pump power, and the escape efficiency.

\subsection{Pound-Drever-Hall (PDH) Control System}

PDH is a control system used to maintain the stability of the laser of the squeezed cavity, which is so sensitive to the environment and the mechanical vibration. PDH consists of an error signal that is proportional to the difference in frequency between the laser light and the cavity resonance. This is done by examining the light reflected from the cavity. Hence, a stable laser frequency may be built by this technique. Therefore, the length of the squeezed cavity can be adjusted through a piezoelectric mounted on the mirror $\mathrm{M}_{\mathbf{S 2}}$. The piezoelectric is controlled electrically as shown in Figure 8.

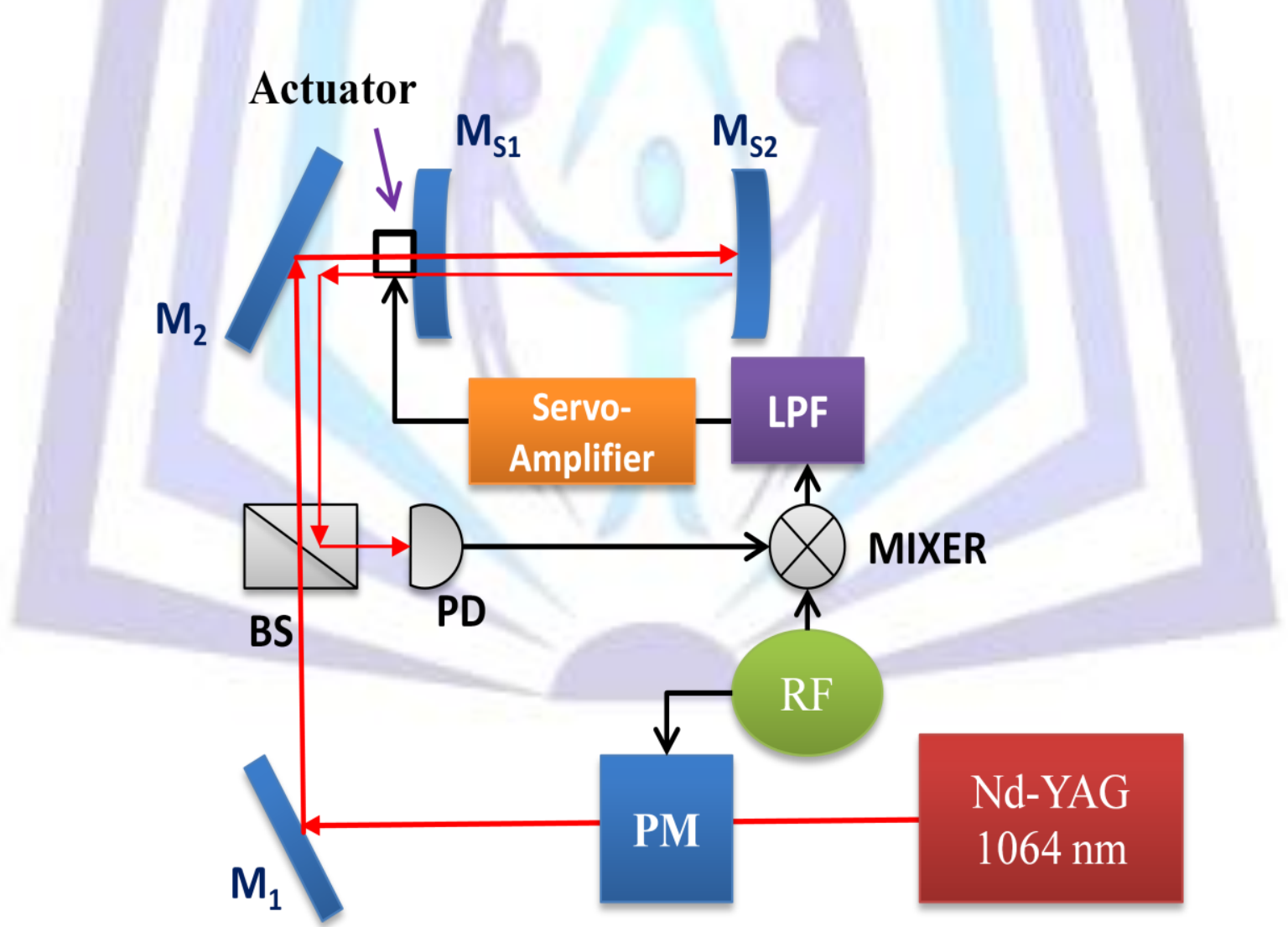

Figure 8: PDH setup. The red dotted line is the optical path while the black solid line is the electrical path. LPF: Low pass filter. BS: Beam splitter. PD: Photodetector. PM: Phase modulator. RF: Radio frequency generator.

From the setup of Figure 8, the laser source is first phase modulated by a nonlinear crystal then the optical-modulated signal is transmitted to the optical resonator (which represents here the squeezed cavity). Next the light beam is reflected back by the mirror $\mathrm{M}_{\mathrm{S} 2}$ and detected by a photodetector. Radio frequency (RF) generator is mixed with the detected signal through an electrical mixer. The mixed signal is used to derive the piezoelectric. In fact all the process of the PDH technique is based on the reflected power of the laser light beam inside the squeezed resonator. 


\subsubsection{PDH Technique}

The idea of this technique is based on the reflected beam. It consists to modulate the phase of the incoming laser beam through a lithium niobate (LNB) crystal. It will result sidebands with a definite phase relationship to the incident and the reflected beam. Then interfere of these sidebands with the reflected beam yields a beat pattern at the modulation frequency. The phase of this pattern will be measured to give an indication on the phase of the reflected beam.

The LNB crystal has a length $L_{s}=1 \mathrm{~cm}$ and width $=5 \mathrm{~mm}$. The voltage is applied transversally on the crystal. The phase modulation depth $\beta$ is equal to [43]

$\beta=-\left(\frac{2 \pi}{\lambda}\right)^{2} \frac{n_{e}{ }^{3}}{2} r_{33} \frac{V_{o}}{d} L$

where $n_{e}=2.156$ at $\lambda=1064 \mathrm{~nm}$ (the extraordinary index of refraction for the LNB crystal), $r_{33}=30 \times 10^{-12} \mathrm{~m} / V$ is the coefficient of the susceptibility matrix, $d=5 \mathrm{~mm}$ is the distance between the two surfaces of the crystal and $V_{o}=25 \mathrm{~V}$ is the applied voltage.

The electric field is phase modulated with a frequency of $\omega_{m}$ by the electro-optical modulator [44]

$e_{\text {in }}=E_{0} \exp \left[i\left(\omega t+\beta \sin \left(\omega_{m} t\right)\right)\right]$

where $\omega_{m}$ is the modulation frequency. From Eqn. (53), the modulation depth $\beta$ is estimated to be -0.004 . Note that $|\beta| \ll 1$ which indicates narrow band phase modulation and therefore the high-order terms in Eqn. (55a) can be neglected

$$
\begin{aligned}
& e_{i n}=E_{0}\left[1+i \beta \sin \left(\omega_{m} t\right)-\frac{\left(\beta \sin \left(\omega_{m} t\right)\right)^{2}}{2 !}+\cdots\right] \exp \left(i \omega_{m} t\right) \\
& e_{i n}=E_{0}\left\{\exp (i \omega t)+\frac{\beta}{2}\left[\exp \left(i\left(\omega+\omega_{m}\right) t\right)-\exp \left(i\left(\omega-\omega_{m}\right) t\right)\right]\right\}
\end{aligned}
$$

Eqn. (55b) displays the carrier at frequency $\omega$ and the two sidebands at frequencies $\left(\omega \mp \omega_{m}\right)$. The reflected beam consists of several reflected sub beams, each one with its appropriate frequency as follow

$$
\begin{aligned}
E_{\text {ref,tot }}= & E_{0}\left\{F(\omega) \exp (i \omega t)+\frac{\beta}{2} F\left(\omega+\omega_{m}\right) \exp \left[i\left(\omega+\omega_{m}\right) t\right]-\right. \\
& \left.\frac{\beta}{2} F\left(\omega-\omega_{m}\right) \exp \left[i\left(\omega-\omega_{m}\right) t\right]\right\}
\end{aligned}
$$

The power in the reflected beam is measured by the photodetector which is proportional to the square of the reflected electric field amplitude. If the power in the carrier is $P_{c}=\left|E_{0}\right|^{2}$, then the power in each sideband is $P_{S}=$ $\left(\beta^{2} / 4\right)\left|E_{0}\right|^{2}$ and the reflected power will be

$$
\begin{aligned}
P_{\text {ref }, \text { tot }=}= & P_{c}|F(\omega)|^{2}+P_{s}\left\{\left|F\left(\omega+\omega_{m}\right)\right|^{2}+\left|F\left(\omega-\omega_{m}\right)\right|^{2}\right\}+ \\
& 2 \sqrt{P_{c} P_{s}}\left\{\operatorname{Re}\left[F(\omega) F^{*}\left(\omega+\omega_{m}\right)-F^{*}(\omega) F\left(\omega-\omega_{m}\right)\right]\right. \\
& \cos \left(\omega_{m} t\right)+\operatorname{Im}\left[F(\omega) F^{*}\left(\omega+\omega_{m}\right)-F^{*}(\omega) F\left(\omega-\omega_{m}\right)\right] \\
& \sin \left(\omega_{m} t\right)+\left(2 \omega_{m} \text { terms }\right)
\end{aligned}
$$

The interesting terms in Eqn. (57) are the oscillating terms at the modulation frequency $\omega_{m}$ because they sample the phase of the reflected carrier. Additionally, $\omega_{m}$ term arises from the interference between the carrier and the sidebands, and $\omega_{m}$ terms come from the sidebands interfering with each other.

\subsubsection{PDH Error Signal Measurement}

The reflected power in Eqn. (57) is measured by the photodetector as shown in Figure 8. Then it mixed with a sinewave signal at the modulation frequency $\omega_{m}$ which is supposed here high frequency about $250 \mathrm{MHz}$. Hence the real part in Eqn. (56) will be vanished and after the LPF only the imaginary term will be remained which represents the error signal $\epsilon$ of the system as indicated in Eqn. (58) [44]

$\epsilon=-\sqrt{P_{c} P_{s}}\left\{\operatorname{Im}\left[F(\omega) F^{*}\left(\omega+\omega_{m}\right)-F^{*}(\omega) F\left(\omega-\omega_{m}\right)\right]\right\}$

\subsection{Homodyne Detection System}

A homodyne detection scheme is proposed in Figure 9 to detect the quadrature squeezed states of the laser light beam. 


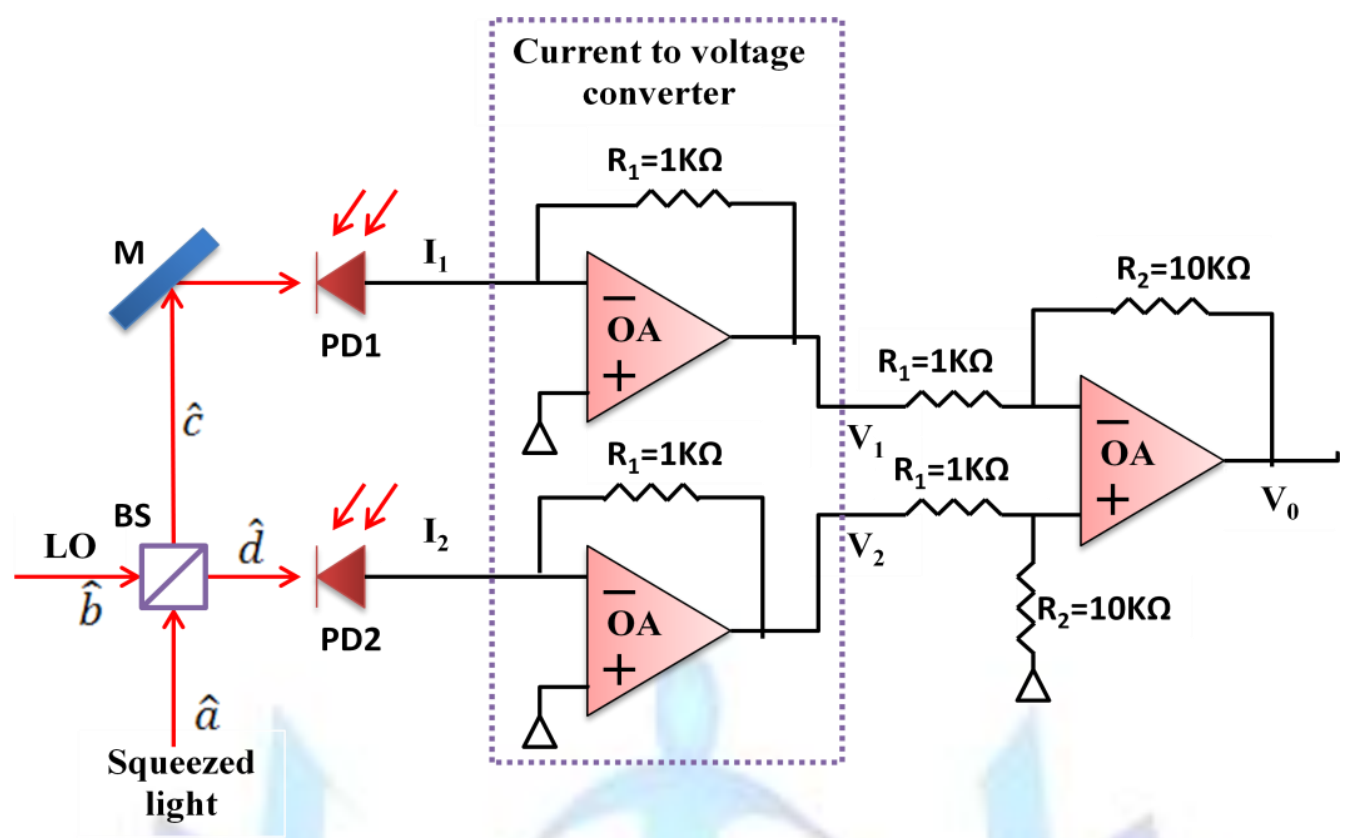

Figure 9: Homodyne scheme to detect quadrature squeezing states of light. M: Mirror. PD1,PD2: Photodetectors. BS: Beam splitter. OA: Operational amplifier. Red solid line: Optical path. Black solid line: Electrical path.

For balanced homodyne detection, the current to voltage converter circuit, $V_{1}$ and $V_{2}$ may be written as

$V_{1}=-R_{1} I_{1}$

$V_{2}=-R_{1} I_{2}$

Thus, the output of the difference amplifier $\mathrm{V}_{0}$ can be expressed as

$$
\begin{aligned}
V_{0} & =\left(V_{2}-V_{1}\right) \frac{R_{2}}{R_{1}} \\
& =-R_{2}\left(I_{2}-I_{1}\right)
\end{aligned}
$$

From Eqn. (60), $V_{0}$ is proportional to $\left(I_{2}-I_{1}\right)$ which is proportional to the difference number of photons $\hat{n}_{2}$ and $\hat{n}_{1}$ [45]

$\left(I_{2}-I_{1}\right) \propto \hat{n}_{1}-\hat{n}_{2}=\hat{n}_{-}$

$\hat{n}_{-}=\hat{a}^{+} \hat{b}+\hat{b}^{+} \hat{a}$

$\hat{a}$ and $\hat{b}$ are the annihilation operators of the coherent state and the LO field, respectively, which may be described by

$\hat{a}=\alpha+\Delta \hat{a}$

$\hat{b}=\beta+\Delta \hat{b}$

Here $\alpha, \beta$ and $\Delta \hat{a}, \Delta \hat{b}$ are the complex amplitudes and the standard deviations of the coherent state $\rho$ and the LO, respectively. Assuming the mode $\hat{b}$ is in the coherent state $\left|\beta e^{-i \omega t}\right\rangle$ with $\beta=|\beta| e^{i \emptyset_{\beta}}$, Eqn. (61b) yields

$\hat{n}_{-}=|\beta|\left(\hat{a}^{+} e^{-i \omega t} e^{i \phi_{\beta}}+\hat{a} e^{-i \omega t} e^{-i \emptyset_{\beta}}\right)$

The squeezed state and the coherent field are derived from the same laser. Thus, they have the same frequency so $\hat{a}=\hat{a}_{0} e^{-i \omega t}$. Then Eqn. (63) can be written as

$$
\begin{aligned}
\hat{n}_{-} & =|\beta|\left(\hat{a}_{0}^{+} e^{i \emptyset_{\beta}}+\hat{a}_{0} e^{-i \emptyset_{\beta}}\right) \\
& =\sqrt{2}|\beta| \Delta \hat{q}_{s}\left(\emptyset_{\beta}\right)
\end{aligned}
$$

The homodyne current is proportional to $\hat{n}_{-}$. Thus, a measurement to the quadrature squeezing is given and the trace of the homodyne measurement can be written as [46]

$Q_{h o m}=\left(\Delta q_{s q}(\Omega)\right)^{2} \cos ^{2} \emptyset_{\beta}+\left(\Delta p_{a n t i s q}(\Omega)\right)^{2} \sin ^{2} \emptyset_{\beta}$

The complete setup to generate squeezed light is shown in Figure 10. 


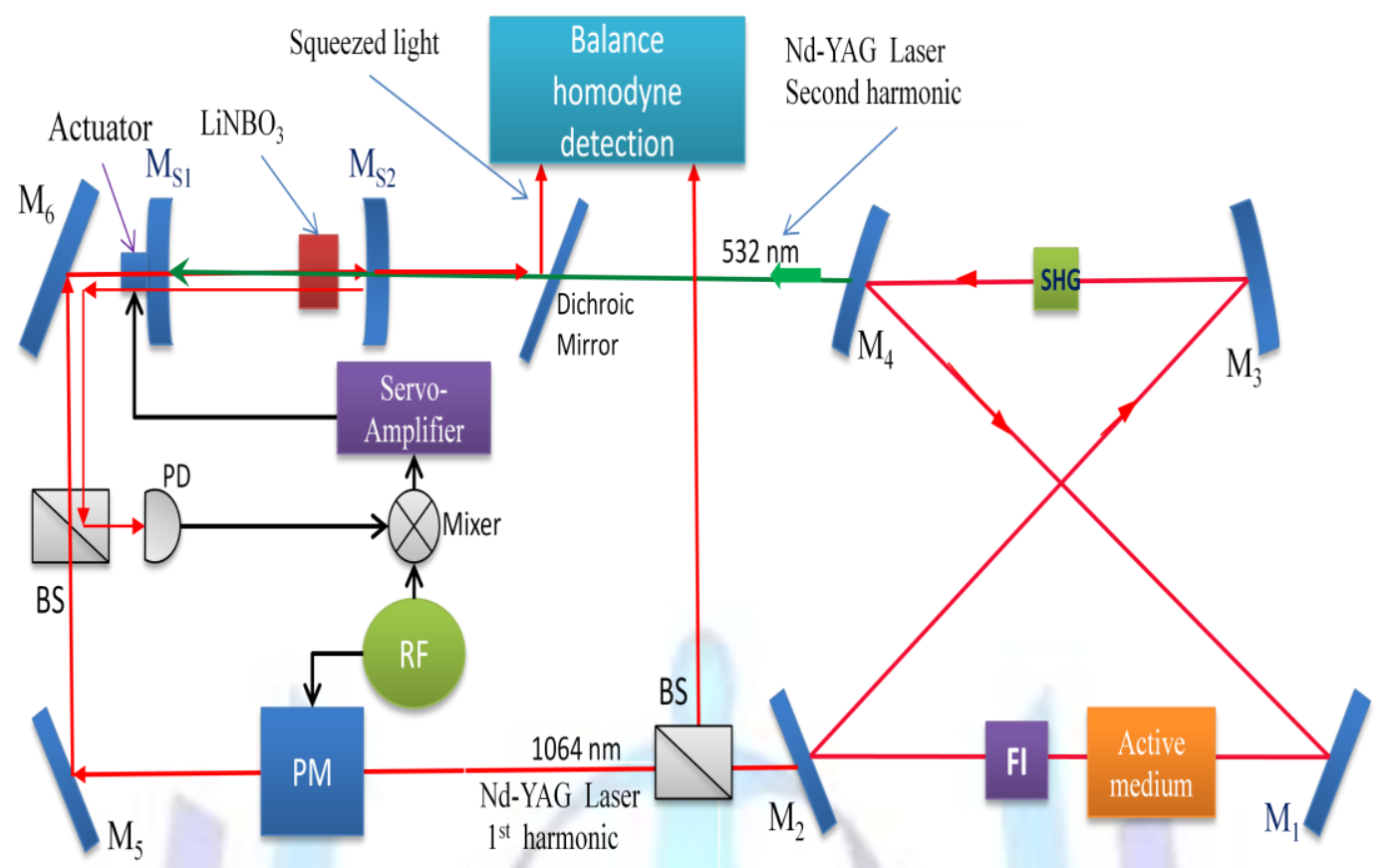

\section{CONCLUSIONS}

Figure 10: Complete setup to generate squeezed light.

Mathematical and experimental frame work has been presented for generating non-classical quantum state using an optical parametric oscillator via a spontaneous parametric down conversion technique. The aim is to investigate squeezed states with quantum noise in one quadrature below the standard quantum limit at the expense of the other. The setup involves four main parts: generation of Nd-YAG second harmonic via a ring resonator, squeezed cavity with a nonlinear crystal inside to generate the squeezed state, Pound-Drever-Hall technique to stabilize the laser in the squeezed cavity and balanced homodyne receiver with high efficiency to detect the squeezed state.

\section{ACKNOWLEGMENT}

Great thanks are gone to the Applied Quantum Mechanics Group in the University of Milano, Italy, especially Prof. Matteo G. A. Paris, Dr. Simone Cialdi, Prof. Fabrizio Catelli and Dr. Stefano Olivares, for their genuine assistant in carrying out the experimental work.

\section{REFERENCES}

[1] I. S. Desher and R. S. Fyath, "Binary quantum communication using squeezed light: Theoretical and experimental Frame Work", International Journal of Computers and Technology, This issue.

[2] J. Binney and D. Skinner, "The physics of quantum mechanics," Capella Archive, 2008.

[3] H. R. Wei and F. G. Deng, "Compact quantum gates on electron-spin qubits assisted by diamond nitrogen-vacancy centers inside cavities," Physical Review A, Vol. 88, No. 4, PP. 42323-42331, October 2013.

[4] M. Cooper, L. J. Wright, C. Soller, and B. J. Smith, "Experimental generation of multi-photon Fock states," Optics Express, Vol. 21, No. 5, PP. 5309-5317, March 2013.

[5] G. Harder, V. Ansari, B. Brecht, Thomas, Dirmeier, C. Marquardt, and C. Silberhorn, "An optimized photon pair source for quantum circuits," Optics Express, Vol. 21, No. 12, PP. 13975-13985, June 2013.

[6] E. d. Valle, "Distilling one, two and entangled pairs of photons from a quantum dot with cavity QED effects and spectral fltering," New Journal of Physics, Vol. 15, No. 2, February 2013.

[7] M. Jofre, A. Gardelein, G. Anzolin, W. Amaya, J. Capmany, R. Ursin, L. Penate, D. Lopez, J. L. San Juan, J. A. Carrasco, F. Garcia, F. J. Torcal-Milla, L. M. Sanchez-Brea, E. Bernabeu, J. M. Perdigues, T. Jennewein, J. P. Torres, M. W. Mitchell, and V. Pruneri, "Fast optical source for quantum key distribution based on semiconductor optical amplifiers," Optics Express, Vol. 19, No. 5, PP. 3825-3834, February 2011.

[8] B. C. Sanders, "Review of Entangled Coherent States," Journal of Physics A, Vol. 45, No. 24, PP. 244002-24023, May 2012.

[9] M.K. Olsen and A.S. Bradley, "Numerical representation of quantum states in the positive-P and Wigner representations," Optics Communications, Vol. 282, No. 19, PP. 3924-3929, October 2009. 
[10] X. Fernandez-Gonzalvo, G. Corrielli, B. Albrecht, M. Grimau, M. Cristiani, and H. de Riedmatten, "Quantum frequency conversion of quantum memory compatible photons to telecommunication wavelengths," Optics Express, Vol. 21, No. 17, PP. 19473-19487, August 2013.

[11] M. Yukawa, K. Miyata, T. Mizuta, H. Yonezawa, P. Marek, R. Filip, and A. Furusawa, "Generating superposition of upto three photons for continuous variable quantum information processing," Optics Express, Vol. 21, No. 5, PP. 5529-5535, March 2013.

[12] R. Prakash, "Use of entangled coherent states in quantum teleportation and entanglement diversion," The First International Workshop on Entangled Coherent States and Its Application to Quantum Information Science | Towards Macroscopic Quantum Communications, PP. 103-110, Tokyo, Japan, 2012.

[13] P. V. Loock, N. Lutkenhaus, W. J. Munro, and Kae Nemoto, "Quantum repeaters using coherent-state communication," Physical Review A, Vol. 78, No. 6, PP. 62319-62329, December 2008.

[14] N. Sangouard, C. Simon, N. Gisin, J. Laurat, R. Tualle-Brouri and P. Grangier, "Quantum repeaters with entangled coherent states," J. Opt. Soc. Am. B, Vol. 27, No. 6, PP. A137-A145, June 2010.

[15] T. Eberle, V. Handchen, and R. Schnabel, "Stable control of $10 \mathrm{~dB}$ two-mode squeezed vacuum states of light," Optics Express, Vol. 21, No. 9, PP. 11546-11553, May 2013.

[16] Y. M. Chi, B. Qi, W. Zhu, L. Qian, H. K. Lo, S. H. Youn, A. I. Lvovsky, and L. Tian, "A balanced homodyne detector for high-rate Gaussian-modulated coherent-state quantum key distribution," New Journal of Physics, Vol. 13, No. 1, PP. 117, January 2011

[17] J. E. Schaar, K. L. Vodopyanov, P. S. Kuo, M. M. Fejer, X. Yu, A. Lin, J. S. Harris, D. Bliss, C. Lynch, V. G. Kozlov, and W. Hurl, "Terahertz Sources Based on Intracavity Parametric Down-Conversion in Quasi-Phase-Matched Gallium Arsenide," IEEE Journal Of Selected Topics In Quantum Electronics, Vol. 14, No. 2, PP. 354-362, March-April 2008.

[18] K. Yu. Spasibko, T. Sh. Iskhakov, and M. V. Chekhova, "Spectral properties of high-gain parametric downconversion," Optics Express, Vol. 20, No. 7, PP. 7507-7515, March 2012.

[19] H. Yonezawa, K. Nagashima, and A. Furusawa, "Generation of squeezed light with a monolithic optical parametric oscillator: Simultaneous achievement of phase matching and cavity resonance by temperature control," Optics Express, Vol. 18, No. 19, PP. 20143-20150, September 2010.

[20] F. Matsuoka, A. Tomita, and A. Okamoto, "Entanglement generation by communication using squeezed states," Physical Review A, Vol. A88, No. 2, PP. 22313-22319, August 2013.

[21] G. Van Assche, "Quantum cryptography and secret-key distillation," Cambridge University Press, 2006.

[22] S. Dwyer, L. Barsotti, S. S. Y. Chua, M. Evans, M. Factourovich, D. Gustafson, T. Isogai, K. Kawabe, A. Khalaidovski, P. K. Lam, M. Landry, N. Mavalvala, D. E. McClelland, G. D. Meadors, C. M. Mow-Lowry, R. Schnabel, R. M. S. Schofield, N. Smith-Lefebvre, M. Stefszky, C. Vorvick, and D. Sigg, "Squeezed quadrature fluctuations in a gravitational wave detector using squeezed light," Optics Express, Vol. 21, No. 16, PP. 19047-19060, August 2013.

[23] H. Grote, K. Danzmann, K. L. Dooley, R. Schnabe, J. Slutsky, and H. Vahlbruch, "First long-term application of squeezed states of light in a gravitational-wave observatory," Physical Review Letter, Vol. 110, No. 18, PP. 181101181105, May 2013.

[24] The LIGO Scientific Collaboration, "A gravitational wave observatory operating beyond the quantum shot-noise limit," Nature Physics, Vol. 7, No. 12, PP. 962-965, September 2011.

[25] K. Tsujino, D. Fukuda, G. Fujii, S. Inoue, M. Fujiwara, M. Takeoka, and M. Sasaki, "Sub-shot-noise-limit discrimination of on-off keyed coherent signals via a quantum receiver with a superconducting transition edge sensor," Optics Express, Vol. 18, No. 8, PP. 8107-8116, April 2010.

[26] K. Tsujino, D. Fukuda, G. Fujii, S. Inoue, M. Fujiwara, M. Takeoka, and M. Sasaki, "Quantum receiver beyond the standard quantum limit of coherent optical communication," Physical Review Letter, Vol. 106, No. 25, PP. 25503-25506, June 2011.

[27] S. Izumi, M. Takeoka, M. Fujiwara, N. D. Pozza, A. Assalini, K. Ema, and M. Sasaki, "Displacement receiver for phase-shift-keyed coherent states," Physical Review Letter, Vol. 86, No. 4, PP. 42328-42339, October 2012.

[28] C. R. Muller, M. A. Usuga, C. Wittmann, M. Takeoka, Ch. Marquardt, U. L. Andersen, and G. Leuchs, "Quadrature phase shift keying coherent state

discrimination via a hybrid receiver," New Journal of Physics, Vol. 14, No. 8, PP. 83009-83020, August 2012.

[29] S. Olivares, S. Cialdi, F. Castelli, and M. G. A. Paris, "Homodyne detection as a near-optimum receiver for phase-shift keyed binary communication in the presence of phase diffusion," Physical Review Letter, Vol. 87, No. 5, PP. 50303-50306, May 2013.

[30] P. Lambropoulos and D. Petrosyan, "Fundamentals of quantum optics and quantum information," Springer-Verlag Berlin Heidelberg, 2007. 
[31] G. Zeng, "Quantum private communication," Higher Education Press, Beijing and Springer-Verlag Berlin Heidelberg, 2010.

[32] S. Olivares, "Quantum optics in the phase space," The European Physical Journal Special Topics, Vol. 203, No. 1, PP. 3-24, April 2012.

[33] Schott data sheet, BK7 sellmeier coefficient. http://www.schott.com.

[34] YAG Brochure data sheet. . http://www.vloc.com.

[35] W. S. C. CHANG, "Principles of lasers and optics," Cambridge University Press, 2005.

[36] O. Gayer, Z. Sacks, E. Galun, and A. Arie1, "Temperature and wavelength dependent refractive index equations for MgO-doped congruent and stoichiometric LiNbO3," Applied Physics B, Vol. 91, No. 4, PP. 343-348, April 2008. (2008).

[37] S. Shi-Kui, Y. Ai-Ying, Z. Lin, C. Jian-Min, and S. Yu-Nan, "Temperature-dependent second-harmonic generation process based on an MgO-doped periodically poled lithium niobate waveguide" Chinese Physics B, Vol. 20, No. 10, PP. 104206-104218, October 2011.

[38] N. Hodgson and H. Weber, "Laser resonator and beam propagation," Springer Science + Business media, Inc., Second Edition, 2005.

[39] K. Thyagarajan and A. Ghatak, "Lasers fundamentals and applications," Springer Science+Business Media, LLC, Second Edition, 2010.

[40] M. Orszag, "Quantum optics including noise reduction, trapped ions,

quantum trajectories, and decoherence," Springer-Verlag Berlin Heidelberg Second Edition, 2008.

[41] S. Dwyer, L. Barsotti, S. S. Y. Chua, M. Evans, M. Factourovich, D. Gustafson, T. Isogai, K. Kawabe, A. Khalaidovski, P. K. Lam, M. Landry, N. Mavalvala, D. E. McClelland, G. D. Meadors, C. M. Mow-Lowry, R. Schnabel, R. M. S. Schofield, N. Smith-Lefebvre, M. Stefszky, C. Vorvick, and D. Sigg, "Squeezed quadrature fluctuations in a gravitational wave detector using squeezed light," Optics Express, Vol. 21, No. 16, PP. 19047-19060, August 2013.

[42] M. Mehmet, H. Vahlbruch, N. Lastzka, K. Danzmann, and R. Schnabel, "Observation of squeezed states with strong photon number oscillations," Physical Review A, Vol. 81, No. 1, PP. 13814-13821, January 2010.

[43] A. Yariv, "Photonics: optical electronics in modern communications," Oxford University Press, Inc., Sixth Edition, 2007.

[44] J. I. Thorpe, K. Numata, and J. Livas, "Laser frequency stabilization andcontrol through offset sideband locking to optical cavities," Optics Express, Vol. 16, No. 20, PP. 15980-15991, September 2008.

[45] F. J. Mendieta, A. Arvizu, R. Muraoka, P. Gallion, J. Sanchez, "Coherent photodetection with applications in quantum communications and cryptography," Proc. of Spie, Vol. 7499, No. 749905-1, 2009.

[46] M. Mehmet, S. Ast, T. Eberle, S. Steinlechner, H. Vahlbruch, and R. Schnabel, "Squeezed light at 1550nm with a quantum noise reduction of 12.3 dB," Optics Express, Vol. 19, No. 25, PP. 25763-25773, December 2011.

\section{Author' biography}

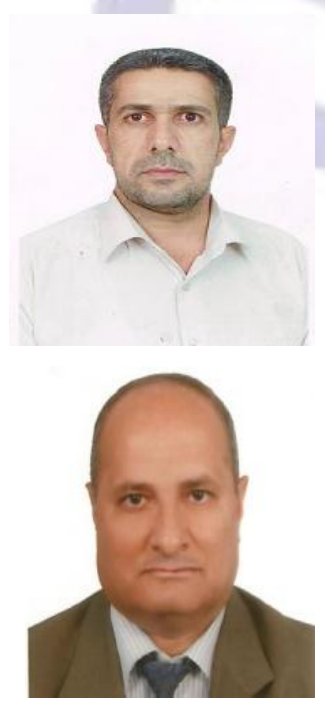

Ismael Shanan Desher was born in Baghdad, Iraq, in 1965. He received the MSc degree in Electrical Engineering from the University of Baghdad, Iraq, in 2007. He joined the staff of the Department of Electrical Engineering, University of Baghdad, as assistant lecturer in 2007. Currently he is working toward the PhD degree in Electronic and Communications engineering at the Institute of Laser for Postgraduate Studies, University of Baghdad. His research interests include quantum communication.

Raad Sami Fyath was born in Maysan, Iraq, in 1954. He received the BSc degree in electrical engineering from the University of Basra, Iraq, in 1976, the MSc degree in electronics and communications engineering from the University of Baghdad, Iraq, in 1987, and the PhD degree in electronics Engineering from University of Wales-Bangor, UK, in 1990. Currently, he is a professor of electronics and communications engineering at the College of Engineering, Alnahrain University, Baghdad, Iraq. His research interests include optical and wireless communications, Optoelectronics, and Nanophotonics. He published more than 100 papers in different scientific journals and conference proceedings. 